\title{
Two-qubit sweet spots for capacitively coupled exchange-only spin qubits
}

\author{
MengKe Feng $\mathbb{1}^{1,2}$, Lin Htoo Zaw $\mathbb{C}^{1,3}$ and Teck Seng Koh $\mathbb{1}^{1 凶}$
}

The implementation of high fidelity two-qubit gates is a bottleneck in the progress toward universal quantum computation in semiconductor quantum dot qubits. We study capacitive coupling between two triple quantum dot spin qubits encoded in the $S=1 / 2, S_{z}=-1 / 2$ decoherence-free subspace-the exchange-only (EO) spin qubits. We report exact gate sequences for CPHASE and CNOT gates, and demonstrate theoretically, the existence of multiple two-qubit sweet spots (2QSS) in the parameter space of capacitively coupled EO qubits. Gate operations have the advantage of being all-electrical, but charge noise that couple to electrical parameters of the qubits cause decoherence. Assuming noise with a $1 / f$ spectrum, two-qubit gate fidelities and times are calculated, which provide useful information on the noise threshold necessary for fault-tolerance. We study two-qubit gates at single and multiple parameter 2QSS. In particular, for two existing EO implementations-the resonant exchange (RX) and the always-on exchange-only (AEON) qubits-we compare two-qubit gate fidelities and times at positions in parameter space where the 2QSS are simultaneously single-qubit sweet spots (1QSS) for the RX and AEON. These results provide a potential route to the realization of high fidelity quantum computation.

npj Quantum Information; https://doi.org/10.1038/s41534-021-00449-4

\section{INTRODUCTION}

Semiconductor quantum dots are one of the leading platforms for building a quantum computer. They present promises of scalability, coherence, and integration with existing microelectronics technologies ${ }^{1,2}$. High fidelity gate operations have been demonstrated in single quantum dot $(\mathrm{QD})^{3,4}$, double $\mathrm{QD}^{5-10}$, and triple quantum dot (TQD) $)^{11-13}$ architectures. In particular, qubits encoded in the decoherence-free subspace of three electron spins $^{14-17}$ have the advantage of fast, all-electrical control. Singlequbit gates are based on the exchange interaction, hence its namesake, the exchange-only (EO) qubit ${ }^{14}$. The total spin of three electrons comprise a $S=3 / 2$ quadruplet and two $S=1 / 2$ degenerate doublets, whose degeneracy can be lifted by an external magnetic field. Logical qubit states are encoded in the total spin $S=1 / 2, S_{z}=-1 / 2$ doublet, which provides immunity against collective decoherence.

The implementation of high fidelity two-qubit gates is a bottleneck in the progress toward universal, fault-tolerant quantum computation. Two-qubit entangling gates can be based on exchange ${ }^{14}$ or capacitive ${ }^{18-21}$ coupling. Exchange is fast but short-ranged, giving rise to hybrid approaches like spinshuttling ${ }^{22-26}$ and circuit QED $^{27,28}$. Because exchange arises from spin-conserving tunneling, two-qubit exchange gates have potential for leakage. On the other hand, capacitive coupling, which arises from electrostatic Coulomb interaction, allows for a longer range of interaction, has less stringent QD addressability requirements, and alleviates the problem of leakage.

We study two capacitively coupled EO qubits, and report exact gate sequences for CPHASE and CNOT gates. The non-local gate is implemented in a single time interval, in contrast to exchange gating which requires several steps ${ }^{14,15,29}$. A major progress in refs. ${ }^{30,31}$ was to propose a single pulse exchange gate, in the negligible transverse coupling limit. Single and two-qubit operations are all-electrical, but charge noise, ubiquitous in the solid-state environment ${ }^{32}$, couple to electrical parameters of the qubits and cause decoherence.

Sweet spots ${ }^{33,34}$ are flat points in the energy landscape which provide protection from parameter fluctuations due to noise. For two existing implementations of the EO qubit-always-on exchange-only $(\mathrm{AEON})^{31}$ and resonant exchange $(\mathrm{RX})^{30,35-38}$ qubits - single-qubit sweet spots (1QSS) have been studied. In ref. ${ }^{31}$, two-qubit sweet spots (2QSS) for exchange coupling was reported. However, sweet spots for capacitive coupling have not been studied, a knowledge gap which we address. We show theoretically, in the weak noise, perturbative limit, that 2QSS exist for single-qubit parameters, $\varepsilon_{\mathrm{m}}, t_{\mathrm{l}}$, and $t_{\mathrm{r}}$, in both control and target qubits. This enables two-qubit gates to be operated at positions in parameter space which are either (1) a single parameter $2 \mathrm{QSS}$, or (2) simultaneously two parameter $2 \mathrm{QSS}$ in $\varepsilon_{\mathrm{m}}$ and $t_{\mathrm{l}}$ (or $t_{\mathrm{r}}$ ), or (3) simultaneously $\varepsilon_{\mathrm{m}} 2 \mathrm{QSS}$ and $\varepsilon 1 \mathrm{QSS}$. For (3), this requires tuning to a particular tunnel coupling ratio, so that the $\varepsilon_{\mathrm{m}}$ 2QSS overlaps with the 1QSS of RX and AEON. Finally, we discuss existence conditions for $2 \mathrm{QSS}$, optimal choices of working points, and address further fidelity optimization.

\section{RESULTS}

\section{Two-qubit Hamiltonian}

We study capacitively coupled EO qubits in a linear array of two TQDs (Fig. 1a). The left/right TQD (qubit $A / B$ ) is the control/target qubit in CPHASE and CNOT gates. QDs are numbered as shown in Fig. 1a. Within each TQD, there are four independently tunable parameters-left/right tunnel couplings $t_{1 / r}$, and detunings for outer and middle QDs, $\varepsilon$ and $\varepsilon_{\mathrm{m}}$. They are defined for qubits $A$ and B: $\varepsilon_{\mathrm{A}} \equiv\left(\varepsilon_{1}-\varepsilon_{3}\right) / 2, \varepsilon_{\mathrm{B}} \equiv\left(\varepsilon_{4}-\varepsilon_{6}\right) / 2, \varepsilon_{\mathrm{mA}} \equiv \varepsilon_{2}-\left(\varepsilon_{1}+\varepsilon_{3}\right) / 2$, and

\footnotetext{
Division of Physics and Applied Physics, School of Physical and Mathematical Sciences, Nanyang Technological University, Singapore, Singapore. ${ }^{2}$ Present address: School of Electrical Engineering and TeleComm., University of New South Wales, Sydney, NSW, Australia. ${ }^{3}$ Present address: Centre for Quantum Technologies, National University of Singapore, Singapore, Singapore. ${ }^{凶}$ email: kohteckseng@ntu.edu.sg
} 
(a)

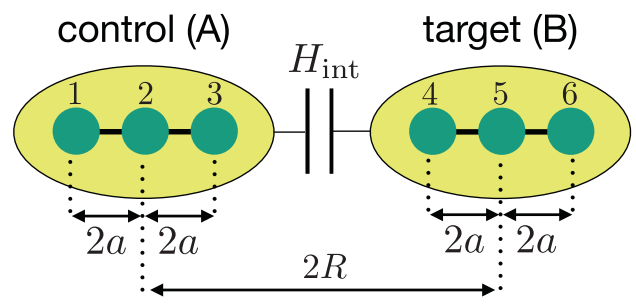

(b)

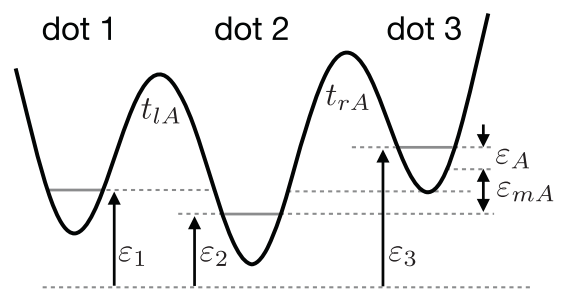

(c)

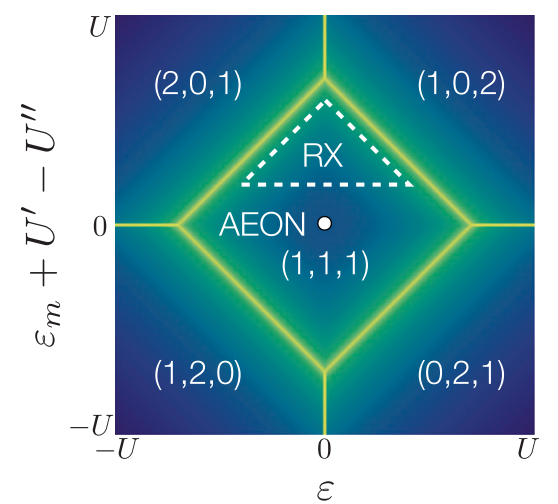

Fig. 1 Exchange-only (EO) qubit parameters. a Two EO qubits in a linear quantum dot (QD) array, with qubit $A(B)$ in QDs 1-3 (4-6). We take $a=50 \mathrm{~nm}, R=160 \mathrm{~nm}$, with QD Bohr radius, $a_{\mathrm{B}}=25 \mathrm{~nm}$ in our calculations. Qubit A (B) is the control (target) qubit in the CPHASE and CNOT gates studied. The capacitive coupling between qubits is given by $\hat{H}_{\text {int }}($ Eq. (6)). b Schematic of parameters in qubit A. Left/ right tunnel couplings are given by $t_{\mathrm{IA} / \mathrm{rA}}$. Detuning parameters $\varepsilon_{i}$ control the relative single-particle energies between $\mathrm{QDs}$, represented by arrows from a reference energy to the ground orbital energy of each QD. Outer and middle detunings, $\varepsilon_{\mathrm{A}} \equiv\left(\varepsilon_{1}-\varepsilon_{3}\right) / 2$ and $\varepsilon_{\mathrm{mA}} \equiv \varepsilon_{2}-\left(\varepsilon_{1}+\varepsilon_{3}\right) / 2$, with the tunnel couplings are sufficient to describe single-qubit dynamics. For all our results, we take a reflection symmetry in the parameters of the two qubits: $\varepsilon_{B}=-\varepsilon_{A}$, $\varepsilon_{\mathrm{mB}}=\varepsilon_{\mathrm{mA}}, t_{\mathrm{IB}}=t_{\mathrm{rA}}$, and $t_{\mathrm{rB}}=t_{\mathrm{IA}}$. c Detuning space of an EO qubit and charge occupation numbers. The AEON single-qubit double ( $\varepsilon$ and $\left.\varepsilon_{\mathrm{m}}\right)$ sweet spot is at the center of the $(1,1,1)$ region at $\left(\varepsilon, \varepsilon_{\mathrm{m}}\right)=$ $\left(0, U^{\prime \prime}-U^{\prime}\right)$ (white circle). The RX single-qubit working region is indicated by the dashed triangle. In our study, the $\mathrm{RX}$ working point is taken to be at $\left(\varepsilon, \varepsilon_{m}\right)=\left(0, U^{\prime}\right)$ (i.e., at its single-qubit $\varepsilon$ sweet spot for equal tunnel couplings).

$\varepsilon_{\mathrm{mB}} \equiv \varepsilon_{5}-\left(\varepsilon_{4}+\varepsilon_{6}\right) / 2$, and schematically represented for qubit $\mathrm{A}$ in Fig. $1 \mathrm{~b}$.

Each EO qubit is fully described by the Hubbard Hamiltonian ${ }^{39}$,

$\hat{\mathcal{H}}=\hat{H}_{\mathrm{t}}+\hat{H}_{\varepsilon}+\hat{H}_{\mathrm{U}}$.

Here, $\hat{H}_{\mathrm{t}}=\sum_{\langle i, j\rangle} \sum_{\sigma} t_{i j} \hat{c}_{i \sigma}^{\dagger} \hat{c}_{j \sigma}$ is nearest-neighbor tunneling, with spin index $\sigma=\{\uparrow, \downarrow\}$ and QD indices $i, j$ run from dots 1-3 (4-6) for qubit $\mathrm{A}$ (B). Tunnel couplings $t_{12} \equiv t_{\mathrm{IA}}, t_{23} \equiv t_{\mathrm{rA}}, t_{45} \equiv t_{\mathrm{IB}}$, and $t_{56} \equiv t_{\mathrm{rB}}$. There is no tunnel coupling between the TQDs, i.e., $t_{34}=0$. The detuning term is $\hat{H}_{\varepsilon}=\sum_{i, \sigma} \varepsilon_{i} \hat{n}_{i \sigma}$, where the number operator $\hat{n}_{i \sigma} \equiv \hat{c}_{i \sigma}^{\dagger} \hat{c}_{i \sigma}$. The Coulomb energy term is $\hat{H}_{U}=\sum_{i} U_{i} \hat{n}_{i \uparrow} \hat{n}_{i \downarrow}+\frac{1}{2} \sum_{i} \sum_{j \neq i} U_{i j} \hat{n}_{i} \hat{n}_{j}$, where the first term is intradot, the second term is inter-dot Coulomb energies, and $\hat{n}_{i}=\hat{n}_{i \uparrow}+\hat{n}_{i \downarrow}$. We consider inter-dot Coulomb energies between all neighbors.

In the perturbative limit $t_{1 / r} \ll U_{i j}<U_{i}$, each EO qubit operates in the $(1,1,1)$ charge state, where numbers represent electron occupation in left, middle, and right dots. This is shown by the central region in detuning space for one qubit in Fig. 1c. However, the encoded logical " 1 " state of the qubit comprises small charge admixtures of doubly occupied charge states $(1,0,2),(2,0,1),(0,2,1)$, and $(1,2,0)$, with a singly-occupied $(1,1,1)$ state. This results in an effective exchange coupling with the encoded logical " 0 " state.

The basis states for qubit $A$ are the singly-occupied encoded qubit states $\left|1_{A}\right\rangle \equiv\left(\left|\downarrow_{1} \downarrow_{2} \uparrow_{3}\right\rangle-\left|\uparrow_{1} \downarrow_{2} \downarrow_{3}\right\rangle\right) / \sqrt{2}$, and $\left|0_{A}\right\rangle \equiv$ $\left(2\left|\downarrow_{1} \uparrow_{2} \downarrow_{3}\right\rangle-\left|\downarrow_{1} \downarrow_{2} \uparrow_{3}\right\rangle-\left|\uparrow_{1} \downarrow_{2} \downarrow_{3}\right\rangle\right) / \sqrt{6}$, and four states comprising a singly- and a doubly-occupied QD residing in the same spin space, $\left|A_{1}\right\rangle \equiv\left|\downarrow_{1} \uparrow_{3} \downarrow_{3}\right\rangle, \quad\left|A_{2}\right\rangle \equiv\left|\uparrow_{1} \downarrow_{1} \downarrow_{3}\right\rangle, \quad\left|A_{3}\right\rangle \equiv\left|\downarrow_{1} \uparrow_{2} \downarrow_{2}\right\rangle$, and $\left|A_{4}\right\rangle \equiv\left|\uparrow_{2} \downarrow_{2} \downarrow_{3}\right\rangle$. Subscripts label QD numbers. Basis states $\left\{\left|0_{B}\right\rangle,\left|1_{B}\right\rangle,\left|B_{i=1 \ldots 4}\right\rangle\right\}$ for qubit $B$ can be written by mapping the QD indices $(1,2,3) \rightarrow(4,5,6)$. With the six-dimensional basis of each qubit, the Schrieffer-Wolff transformation ${ }^{40-42}$ gives effective single-qubit Hamiltonians,

$\hat{H}_{\text {eff }, \mathrm{A}}=-\frac{\hbar \omega_{\mathrm{A}}}{2} \hat{\sigma}_{z}-\frac{\hbar g_{\mathrm{A}}}{2} \hat{\sigma}_{x}$,
$\hat{H}_{\mathrm{eff}, \mathrm{B}}=-\frac{\hbar \omega_{\mathrm{B}}}{2} \hat{\sigma}_{z}-\frac{\hbar g_{\mathrm{B}}}{2} \hat{\sigma}_{x}$,

where $\hbar \omega \equiv 2\left(t_{1}^{2} b_{-}+t_{r}^{2} b_{+}\right), \quad \hbar g \equiv 2 \sqrt{3}\left(t_{1}^{2} b_{-}-t_{r}^{2} b_{+}\right), \quad$ and $b_{ \pm} \equiv 1 /\left(U-U^{\prime \prime} \pm \varepsilon+\varepsilon_{\mathrm{m}}\right)+1 /\left(U-2 U^{\prime}+U^{\prime \prime} \mp \varepsilon-\varepsilon_{\mathrm{m}}\right)$. (Qubit subscripts $A, B$ have been omitted for brevity here.) In the derivation, we assumed identical QDs: $U_{i} \equiv U$ for all $i, U_{12}=U_{23}=$ $U_{45}=U_{56} \equiv U^{\prime}$ and $U_{13}=U_{46} \equiv U^{\prime \prime}$ (for numerical values, see "Methods"). Encoded states, dressed by charge admixtures, and denoted with a prime, are

$$
\begin{aligned}
& \left|1_{\mathrm{A}}^{\prime}\right\rangle=\frac{1}{\sqrt{N_{\mathrm{A}}}}\left(\left|1_{\mathrm{A}}\right\rangle+\sum_{i=1}^{4} a_{i}\left|\mathrm{~A}_{i}\right\rangle\right), \\
& \left|1_{\mathrm{B}}^{\prime}\right\rangle=\frac{1}{\sqrt{N_{\mathrm{B}}}}\left(\left|1_{\mathrm{B}}\right\rangle+\sum_{i=1}^{4} \beta_{i}\left|\mathrm{~B}_{i}\right\rangle\right),
\end{aligned}
$$

where $\sqrt{N_{\mathrm{A} / \mathrm{B}}}$ are normalization constants. Admixtures $a_{i,} \beta_{i}$ depend on electrical parameters $t_{\mathrm{l} / \mathrm{r}}, \varepsilon_{\text {, and }} \varepsilon_{\mathrm{m}}$, which are detailed in Supplementary Method 1.

Two-qubit capacitive coupling arises from the inter-dot Coulomb interaction between the TQDs, $\hat{H}_{\text {int }}=\sum_{i=1}^{3} \sum_{j=4}^{6} \mathcal{V}_{i j} \hat{n}_{i} \hat{n}_{j}$. We denote inter-TQD Coulomb terms by $\mathcal{V}_{i j}$ to distinguish them from intra-TQD terms $U_{i j}$. This distinction is for notational clarity; the physical basis-electrostatic interaction-is the same. In the computational basis $\left\{\left|0_{A} 0_{B}\right\rangle,\left|0_{A} 1_{B}^{\prime}\right\rangle,\left|1_{A}^{\prime} 0_{B}\right\rangle,\left|1_{A}^{\prime} 1_{B}^{\prime}\right\rangle\right\}$, the twoqubit capacitive coupling is diagonal, given by

$\hat{H}_{\text {int }}=\hbar \times \operatorname{diag}\left\{\mathrm{V}_{1}, \mathrm{~V}_{2}, \mathrm{~V}_{3}, \mathrm{~V}_{4}\right\}$,

which comprises a global phase, single-qubit energy shifts, and a $\hat{\sigma}_{z} \otimes \hat{\sigma}_{z}$ term equivalent to the Ising (ZZ) model (see Supplementary Note 1). Tri-quadratic confinement potentials (see "Methods") provide analytical expressions for $V_{i}$ terms (see Supplementary Methods 2, 3). The analytical expressions are crucial for the calculation of 2QSS and gate fidelities, explained in the rest of this paper. Finally, the full two-qubit Hamiltonian in the computational basis is

$\hat{H}=\hat{H}_{\text {eff, } \mathrm{A}} \otimes \hat{\mathbb{1}}_{\mathrm{B}}+\hat{1}_{\mathrm{A}} \otimes \hat{H}_{\text {eff } \mathrm{B}}+\hat{H}_{\text {int }}$.

Because capacitive coupling arises from the overlap of qubit wavefunctions, by increasing the barrier between QD 3 and 4, the exponential "tail" of the wavefunctions can be arbitrarily reduced, thereby turning off the interaction. Thus, energy shifts from the presence of the other qubit (equivalent to a redefinition of detunings) does not affect $1 \mathrm{QSS}$ and single-qubit gates. This redefinition shifts 
the entire charge boundary diagram, and hence 1QSS (Fig. 1c) by an approximately constant amount, $\Delta \varepsilon=0.54 \mathrm{meV}, \Delta \varepsilon_{\mathrm{m}}=0.21 \mathrm{meV}$, for parameters used in our study.

The $\hbar V_{i}$ terms in Eq. (6) contain all pairwise inter-dot Coulomb energies between the two TQDs, weighted by the charge admixtures. The latter is key in modeling the effect of charge noise, which has been measured to be $1 / f$-like over a wide range of frequencies ${ }^{3,32,43,44}$.

\section{Charge noise}

We introduce noise by simulating random fluctuations in tunneling and detuning ${ }^{45,46}$. These fluctuations perturb charge admixtures, leading to noisy two-qubit interaction. At this point, we write qubit parameters in vectorized form $\boldsymbol{n}=\left(t_{\mathrm{IA}}, t_{\mathrm{rA}}, \varepsilon_{\mathrm{A}}, \varepsilon_{\mathrm{mA}}\right.$, $\left.t_{\mathrm{BB}}, t_{\mathrm{rB}}, \varepsilon_{\mathrm{B}}, \varepsilon_{\mathrm{mB}}\right)$ for notational simplicity, where the first (last) four components belong to qubit $A(B)$. Noisy parameters, denoted with a tilde, is the sum of the noiseless parameter with a random time-dependent fluctuation, $\tilde{n}_{i}(t)=n_{i}+\delta n_{i}(t)$. We assume uncorrelated noise and each random time series is generated independently in simulations. To avoid confusion, symbol $t$ with or without numeral subscripts indicates time, while $t_{1 / r, A / B}$ indicate left/right tunnel couplings for qubits $A / B$. It should be clear from the context which is being referred to. Random variables are characterized by the time correlation function $C_{n_{i}}\left(t_{1}-t_{2}\right)=$ $\left\langle\delta n_{i}\left(t_{1}\right) \delta n_{i}\left(t_{2}\right)\right\rangle$, where angular brackets $\langle$.$\rangle denote average over$ noise realizations. The corresponding (two-sided) power spectral density is the Fourier transform of the time correlation function, $S_{n_{i}}(\omega)=\int_{-\infty}^{\infty} C_{n_{i}}(t) \mathrm{e}^{-i \omega t} d t$. Adapting the algorithms of refs. ${ }^{47,48}$, each noisy parameter $\delta n_{i}(t)$ is simulated with a $1 / f$ power spectral density with a low-frequency roll-off,

$S_{n_{i}}(\omega)=\left\{\begin{array}{cl}\Delta_{n_{i}}^{2} \frac{2 \pi}{\omega_{1}}, & \text { for }|\omega| \leq \omega_{1} \\ \Delta_{n_{i}}^{2} \frac{2 \pi}{|\omega|}, & \text { for } \omega_{1} \leq|\omega| \leq \omega_{\mathrm{h}} \\ 0, & \text { otherwise }\end{array}\right.$

where $\omega_{1}\left(\omega_{\mathrm{h}}\right)$ is the lower (higher) cutoff frequency. $\Delta_{n_{i}}^{2}$ is related to the noise variance $\sigma_{n_{i}}^{2}$ by $\Delta_{n_{i}}^{2}=\sigma_{n_{i}}^{2} \frac{1}{2}\left(1+\ln \left(\omega_{\mathrm{h}} / \omega_{1}\right)\right)^{-1}$ (see Supplementary Note 2). Although spectroscopy experiments ${ }^{3,43,44}$, which are limited by acquisition time, have not observed lowfrequency saturation in $1 / f$ noise, we model the roll-off to avoid unphysical, infinite noise power at $f=0$ (See Supplementary Discussion 1).

Because our objective is to study fidelities and sweet spots of two-qubit gates under noise, and 1QSS have already been found, we assume noisy two-qubit interactions and ideal (noiseless) single-qubit operations for all calculations.

\section{Non-local gate time}

CPHASE and CNOT gates are given by unitary evolution operators

$\hat{U}_{\mathrm{CPHASE}}=\left(\begin{array}{cccc}1 & 0 & 0 & 0 \\ 0 & 1 & 0 & 0 \\ 0 & 0 & 1 & 0 \\ 0 & 0 & 0 & -1\end{array}\right), \hat{U}_{\mathrm{CNOT}}=\left(\begin{array}{llll}1 & 0 & 0 & 0 \\ 0 & 1 & 0 & 0 \\ 0 & 0 & 0 & 1 \\ 0 & 0 & 1 & 0\end{array}\right)$.

Using Mahklin invariants ${ }^{49}$, we find the non-local interaction time required for these gates to be identical,

$t_{0}=k \pi /\left[\left(V_{1}+V_{4}\right)-\left(V_{2}+V_{3}\right)\right]$,

for any odd positive integer $k$ (see Supplementary Note 3 ). While non-local gating has been studied in the literature $30,31,36,50,51$, the exact gate sequence (including local gates) for capacitively coupled EO qubits has not been reported, to our knowledge. We show the exact sequence and timings in Fig. 2a. Importantly, it is the energy difference in the denominator $\left(V_{1}+V_{4}\right)-\left(V_{2}+V_{3}\right)$, that is important for capacitive gating. This difference is dominated by Coulomb interactions between electrons in doubly-occupied charge admixtures of qubits $A$ and $B$, which have a center of mass situated away from the middle dot (unlike the dominant $(1,1,1)$ configuration), giving rise to a net electric dipole moment. The energy difference can be qualitatively understood as a dipole-dipole interaction between the two TQDs. This is what gives rise to the dependence on TQD parameters for the non-local gate time.

Color plots of gate time $t_{0}$ in detuning space for qubit $A$ are shown, for equal tunneling ratios where $\arctan \left(t_{\mathrm{IA}} / t_{\mathrm{rA}}\right)=45^{\circ}$ (Fig. 2b) and tunneling ratios given by $\arctan \left(t_{\mathrm{IA}} / t_{\mathrm{rA}}\right)=57^{\circ}$ (Fig. 2e). The equivalent color plot for qubit $B$ (not shown) is a mirror reflection about the $\varepsilon=0$ line for qubit $A$, because of our choice of parameters $\varepsilon_{\mathrm{B}}=-\varepsilon_{\mathrm{A}}$ and $t_{\mathrm{IA} / \mathrm{B}}=t_{\mathrm{rB} / \mathrm{A}}$. Gate times are faster near the boundaries of $(1,1,1)$ with $(2,0,1)$ and $(1,0,2)$ charge occupations. This can be understood as a stronger capacitive interaction arising from a larger mean dipole moment of each TQD as a result of proportionately larger charge admixtures. Comparatively, sitting near $(1,2,0)$ or $(0,2,1)$ boundaries gives rise to a smaller net TQD dipole moment and thus a significantly longer gate time. In addition, for qubit $A$, gate times decrease faster as one goes from the central $(1,1,1)$ region toward the $(1,0,2)$ boundary, compared to moving toward the $(2,0,1)$ boundary. This is because of the stronger dipole-dipole interaction arising from proximity of the doubly occupied $(1,0,2)$ state of qubit $A$ with $B$. The converse is true for qubit $B$, i.e., gate times decrease faster moving from the central region toward the $(2,0,1)$ boundary, compared to the $(1,0,2)$ boundary.

The AEON works at the $\varepsilon=0, \varepsilon_{\mathrm{m}}=U^{\prime \prime}-U^{\prime}=-0.9 \mathrm{meV}$ double $1 \mathrm{QSS}{ }^{31}$, and is independent of tunnel coupling. The RX operates within the upper triangular region (Fig. 1c) ${ }^{35,37}$. We take the RX operating point to be at $\varepsilon=0$ (which is a 1QSS for symmetric tunnel coupling), and $\varepsilon_{\mathrm{m}}=-0.57 \mathrm{meV}$. Asymmetric tunnel couplings shift the RX $\varepsilon$ SS. We assume tunnel couplings are tunable; they can be tuned to the desired ratio, if necessary, during nonlocal gating and back to equal tunnel coupling (for RX SS) during single-qubit operations. Consequently, the fastest $(k=1)$ non-local gate times for RX and AEON are 64 and $450 \mathrm{~ns}$, respectively, for numerical parameters used. The choice of $\varepsilon_{\mathrm{m}}$ for RX is slightly arbitrary; it is possible for the RX gate time to be faster with larger $\varepsilon_{\mathrm{m}}$, thereby moving the operating point closer to the doubly occupied regime while remaining in the $(1,1,1)$ configuration. The trade-off is greater susceptibility to charge noise.

\section{Two-qubit gate fidelity}

Noisy two-qubit evolution given by

$\tilde{U}_{\text {int }}\left(t_{0}\right)=\exp \left\{-\frac{\mathrm{i}}{\hbar} \int_{0}^{t_{0}} \tilde{H}_{\text {int }}\left(t^{\prime}\right) d t^{\prime}\right\}$

can be decomposed into ideal and noisy evolution,

$$
\begin{aligned}
& \tilde{U}_{\text {int }}\left(t_{0}\right)=\hat{U}_{\text {int }}\left(t_{0}\right) \hat{U}_{\delta}\left(t_{0}\right) \\
& =\left(\begin{array}{cccc}
\mathrm{e}^{-i V_{1} t_{0}} & 0 & 0 & 0 \\
0 & \mathrm{e}^{-i V_{2} t_{0}} & 0 & 0 \\
0 & 0 & \mathrm{e}^{-\mathrm{i} V_{3} t_{0}} & 0 \\
0 & 0 & 0 & \mathrm{e}^{-i V_{4} t_{0}}
\end{array}\right)\left(\begin{array}{cccc}
\mathrm{e}^{-\mathrm{i} \int_{0}^{t_{0}} \delta V_{1}\left(t^{\prime}\right) d t^{\prime}} & 0 & 0 & 0 \\
0 & \mathrm{e}^{-\mathrm{i} \int_{0}^{t_{0}} \delta V_{2}\left(t^{\prime}\right) d t^{\prime}} & 0 & 0 \\
0 & 0 & \mathrm{e}^{-\mathrm{i} \int_{0}^{t_{0}} \delta V_{3}\left(t^{\prime}\right) d t^{\prime}} & 0 \\
0 & 0 & 0 & \mathrm{e}^{-\mathrm{i} \int_{0}^{t_{0}} \delta V_{4}\left(t^{\prime}\right) d t^{\prime}}
\end{array}\right) .
\end{aligned}
$$

We use the two-qubit gate fidelity ${ }^{52}, \mathcal{F}=\frac{1}{d^{2}}\left|\operatorname{Tr}\left(U^{\dagger} \tilde{U}\right)\right|^{2}$, where $d$ is dimensionality, to evaluate the effect of charge noise. We compute the gate fidelity for exact CPHASE and CNOT gate sequences (Fig. 2a), averaged over noisy two-qubit interactions. Since CPHASE and CNOT gates share the same non-local interaction, the fidelity expression for both are 
(a)

Exact CNOT

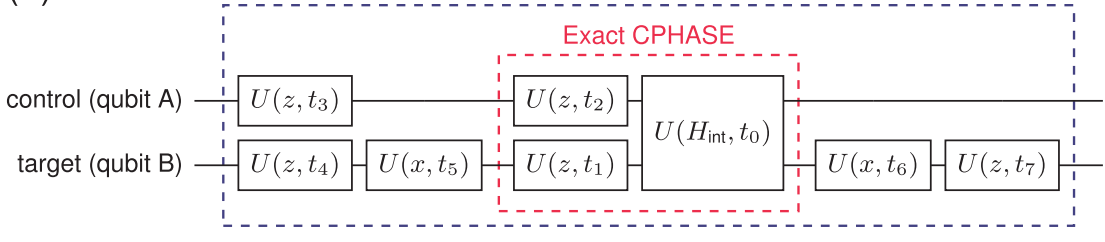

$t_{0}=k \pi /\left(V_{1}-V_{2}-V_{3}+V_{4}\right) \quad t_{1}=t_{0}\left(V_{1}-V_{2}\right) / \omega_{B} \quad t_{2}=t_{0}\left(V_{1}-V_{3}\right) / \omega_{A} \quad t_{3}=2 \pi / \omega_{A}$

$t_{4}=\pi /\left(2 \omega_{B}\right) \quad t_{5}=\pi /\left(2 g_{B}\right) \quad t_{6}=3 \pi /\left(2 g_{B}\right) \quad t_{7}=3 \pi /\left(2 \omega_{B}\right)$

\section{$\arctan \left(t_{l A} / t_{r A}\right)=45^{\circ}$}

(b)

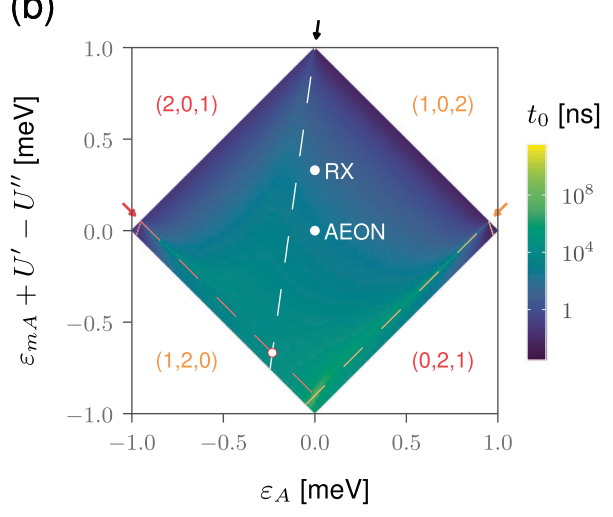

(c)
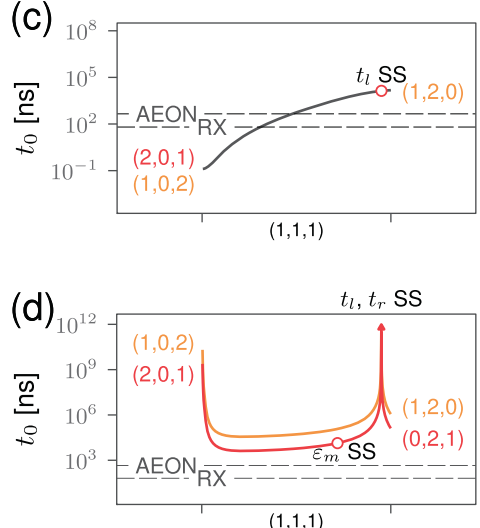
$\underline{\arctan \left(t_{l A} / t_{r A}\right)=57^{\circ}}$

(e)

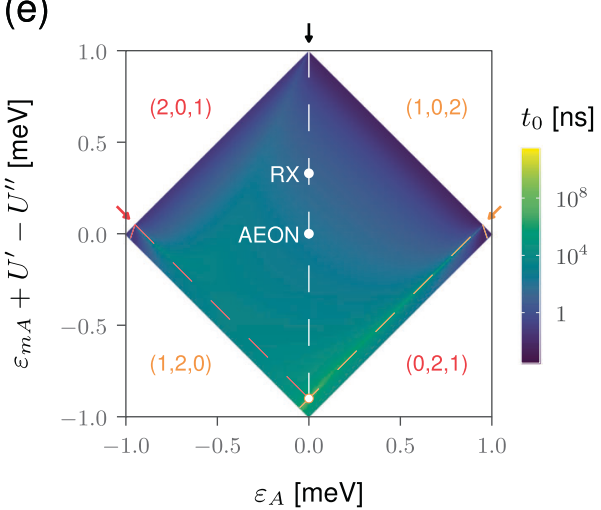

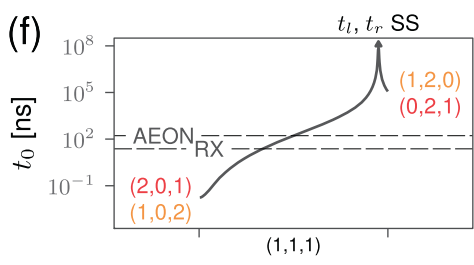

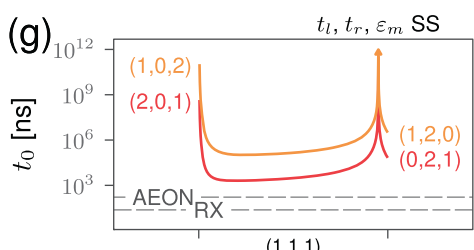

Fig. 2 Gate sequences and gate times in detuning space. a Exact gate sequences and timings for CPHASE (red dashed box) and CNOT (blue dashed box). Effective single-qubit Hamiltonians (Eqs. (2), (3)) generate unitaries for qubit $\mathrm{A} / \mathrm{B}, U_{\mathrm{A} / \mathrm{B}}(x, t) \equiv \exp \left(\mathrm{i} \hat{\sigma}_{x} g_{\mathrm{A} / \mathrm{B}} t / 2\right)$ and $U_{\mathrm{A} / \mathrm{B}}(z, t) \equiv \exp \left(\mathrm{i} \hat{\sigma}_{z} \omega_{\mathrm{A} / \mathrm{B}} t / 2\right)$. The non-local unitary is given by Eq. (11) without noise. The non-local gate time $t_{0}$ is identical for CPHASE and CNOT, where $k$ is an odd positive integer (Eq. (10)). b Color plot of gate times for equal tunneling $t_{\mathrm{IA}}=t_{\mathrm{rA}}$, with two-qubit sweet spots (2QSS) indicated for $\varepsilon_{\mathrm{mA}}$ (white), $t_{\mathrm{IA}}$ (red), and $t_{\mathrm{rA}}$ (orange) with dashed lines. c Linecuts of $\varepsilon_{\mathrm{mA}} 2 \mathrm{QSS}$. Fastest gate times are at the top corner and increase down the $\varepsilon_{\mathrm{mA}} 2 \mathrm{QSS}$ line. Fastest $(k=1) \mathrm{AEON}$ and RX gate times are 450 and $64 \mathrm{~ns}$, respectively. At the intersection with $t_{\mathrm{IA}} 2 \mathrm{QSS}$, gate time is $13.6 \mu \mathrm{s}$. d Linecuts of $t_{\mathrm{IA}}$ and $t_{\mathrm{rA}} 2 \mathrm{QSS}$. Gate times go to infinity at the point where the two tunneling $2 \mathrm{QSS}$ intersect. Panels (e-g) repeat panels $(\mathbf{b}-\mathbf{d})$, but with tunneling $\arctan \left(t_{\mathrm{IA}} / t_{\mathrm{rA}}\right)=57^{\circ}$, chosen such that the $\varepsilon_{\mathrm{m}} 2 \mathrm{QSS}$ is along $\varepsilon_{\mathrm{A}}=0$, which is the $1 \mathrm{QSS}$, and which the operating points of AEON and RX lie on. As before, gate times increase as $\varepsilon_{\mathrm{mA}}$ decreases, and goes to infinity at the $t_{\mathrm{IA}}, t_{\mathrm{rA}}$ double $2 \mathrm{QSS}$. 
identical. The average gate fidelity ${ }^{53}$ is

$$
\begin{aligned}
\langle\mathcal{F}\rangle & =\frac{1}{16}\left\langle\left|\operatorname{Tr}\left(\tilde{U}_{\delta}\left(t_{0}\right)\right)\right|^{2}\right\rangle \\
& =\frac{1}{16} \sum_{i, j}\left\langle\mathrm{e}^{-\mathrm{i} \int_{0}^{t_{0}} \mathrm{~d} t^{\prime}\left(\delta V_{i}\left(t^{\prime}\right)-\delta V_{j}\left(t^{\prime}\right)\right)}\right\rangle .
\end{aligned}
$$

This formula is used for all our numerical simulations. We note that this formula is identical to process fidelity ${ }^{54}$; the averaging over noise realizations done in our numerical simulations and analytical calculations correspond to the measurement protocols performed in quantum process tomography experiments (see Supplementary Note 4).

\section{Analytical fidelity formula}

Next, we derive an approximate analytical expression for average gate fidelity. Assuming stationary, Gaussian noise with zero mean, and making use of the series expansion to linear order, $\delta V_{i}=\sum_{j} \frac{\partial V_{i}}{\partial n_{j}} \delta n_{j}$, we perform a cumulant expansion ${ }^{55,56}$ of Eq. (13) to obtain an analytical expression for average gate fidelity,

$$
\begin{aligned}
\left\langle\mathcal{F}_{\text {an }}\right\rangle= & \frac{1}{4}\left(1+\exp \left\{-\frac{1}{2} \sum_{i=1}^{4}\left(\left(\boldsymbol{F}_{\mathrm{A}}-C_{11} \mathbf{1}\right)^{\top} \frac{\partial \boldsymbol{\xi}}{\partial n_{i}}\right)^{2} \sigma_{n_{i}}^{2} \varsigma\left(t_{0}\right)\right\}\right) \\
& \times\left(1+\exp \left\{-\frac{1}{2} \sum_{i=5}^{8}\left(\left(\boldsymbol{F}_{\mathrm{B}}-C_{11} \mathbf{1}\right)^{\top} \frac{\partial \boldsymbol{v}}{\partial n_{i}}\right)^{2} \sigma_{n_{i}}^{2} \varsigma\left(t_{0}\right)\right\}\right) .
\end{aligned}
$$

The derivation is detailed in Supplementary Note 5 . Here $\varsigma\left(t_{0}\right)=\int_{0}^{t_{0}} \mathrm{~d} t^{\prime} \int_{0}^{t^{\prime}} \mathrm{d} t^{\prime \prime} \bar{C}\left(t^{\prime \prime}\right)$ is the double integral of the noise correlation function, normalized to $\bar{C}(0)=1$, and $\sigma_{n_{i A \mathrm{~B}}}$ is the noise standard deviation in first/last four $\boldsymbol{n}$ components for qubit $A / B$. The terms $\boldsymbol{F}_{\mathrm{A}}, \boldsymbol{F}_{\mathrm{B}}, \boldsymbol{\xi}, \boldsymbol{v}$ comprise linear combinations of Coulomb integrals and are detailed in Supplementary Method 3. The term $C_{11} \equiv \sum_{i=1}^{3} \sum_{j=1}^{3} \mathcal{V}_{i j}$ comprises all possible pairwise Coulomb energies between electrons of qubits $A$ and $B$ in the $(1,1,1)_{A}-$ $(1,1,1)_{B}$ configuration. The function $\varsigma\left(t_{0}\right)$ is general; it can be obtained from any noise power spectrum. In this study, $\varsigma\left(t_{0}\right)$ is calculated from the $1 / f$ power spectrum in Eq. (8). The exact expression is given by Supplementary Eq. (101) and its derivation shown in Supplementary Note 2.

\section{Two-qubit sweet spots (2QSS)}

Simplification of the noise terms of Eq. (14) yields insights into the existence of $2 \mathrm{QSS}$. A $2 \mathrm{QSS}$ is defined to be a point in parameter space for which all partial derivatives, $\partial V_{i} / \partial n_{j}=0$, for a particular parameter $n_{j}$. At these points, each qubit is protected from noise in parameter $n_{j}$. First, interaction terms $V_{i}$ for $i=2,3,4$, contain charge admixtures through the $\left|1_{\mathrm{A} / \mathrm{B}}^{\prime}\right\rangle$ state; these admixtures depend on TQD parameters which couple to charge noise. This means $\delta V_{1}=0$ because the $\left|0_{A} 0_{B}\right\rangle$ state does not contain admixtures. Second, $\left|0_{A} 1_{B}^{\prime}\right\rangle$ and $\left|1_{A}^{\prime} 0_{B}\right\rangle$ states contain admixtures in qubits $B$ and $A$, respectively, implying that $\delta V_{2}$ and $\delta V_{3}$ are only dependent on noise in the respective qubits. Explicitly evaluating the expansions $\delta V_{2} \approx \sum_{j} \frac{\partial V_{2}}{\partial n_{j}} \delta n_{j}=\frac{\partial V_{2}}{\partial \varepsilon_{B}} \delta \varepsilon_{\mathrm{B}}+\frac{\partial V_{2}}{\partial \varepsilon_{\mathrm{mB}}} \delta \varepsilon_{\mathrm{mB}}+\frac{\partial V_{2}}{\partial t_{\mathrm{B}}} \delta t_{\mathrm{IB}}+\frac{\partial V_{2}}{\partial t_{\mathrm{rB}}} \delta t_{\mathrm{rB}}$, and $\delta V_{3} \approx \sum_{j} \frac{\partial V_{3}}{\partial n_{j}} \delta n_{j}=\frac{\partial V_{3}}{\partial \varepsilon_{A}} \delta \varepsilon_{A}+\frac{\partial V_{3}}{\partial \varepsilon_{m A}} \delta \varepsilon_{m A}+\frac{\partial V_{3}}{\partial t_{A}} \delta t_{1 A}+\frac{\partial V_{3}}{\partial t_{\mathrm{rA}}} \delta t_{\mathrm{rA}}$, we indeed find that noise in qubit $B(A)$ affects only $\delta V_{2}\left(\delta V_{3}\right)$. In truncating the series expansions to terms linear in fluctuating parameters, we have assumed the weak noise limit. In addition, we keep only leading order terms within the partial derivatives in the perturbative limit. As a result, $\delta V_{4} \approx \delta V_{2}+\delta V_{3}$. These approximations allow us to separate the contributions of noise in the two qubits. Explicit forms of the derivatives are provided in Supplementary Note 5 .

The locus of $2 \mathrm{QSS}$ is shown as dashed lines in detuning space of qubit $A$ in Fig. $2 b$, e. As described in the preceding section, equivalent color plots for qubit $B$ are reflections about the $\varepsilon=0$ line, and the discussion about qubit $A$ here also applies to qubit $B$. The $\varepsilon_{\mathrm{mA}} 2 \mathrm{QSS}$ for qubit $A$ is indicated as the white dashed line in Fig. $2 b$, e. The angle of the $\varepsilon_{\mathrm{mA} / \mathrm{B}} 2 \mathrm{QSS}$ line depends on tunnel coupling ratio, and equations of the lines are

$$
\left.\frac{t_{I A}}{t_{r A}}=\sqrt{\frac{C_{51}-C_{11}}{C_{21}-C_{11}}}\left(\frac{U-2 U^{\prime}+U^{\prime \prime}-\varepsilon_{m A}-\varepsilon_{A}}{U-2 U^{\prime}+U^{\prime \prime}-\varepsilon_{m A}+\varepsilon_{A}}\right)^{3 / 2}, \quad \text { ( } \varepsilon_{m A} 2 \mathrm{QSS}\right),
$$

$\frac{t_{/ B}}{t_{r B}}=\sqrt{\frac{C_{13}-C_{11}}{C_{14}-C_{11}}}\left(\frac{U-2 U^{\prime}+U^{\prime \prime}-\varepsilon_{m B}-\varepsilon_{B}}{U-2 U^{\prime}+U^{\prime \prime}-\varepsilon_{m B}+\varepsilon_{B}}\right)^{3 / 2}, \quad\left(\varepsilon_{\mathrm{mB}}\right.$ 2QSS $)$,

Here, $C_{11}, C_{51}, C_{21}, C_{13}$, and $C_{14}$ comprise all pairwise Coulomb energies between electrons of the TQDs for $(1,1,1)_{A}-(1,1,1)_{B}$, $(0,2,1)_{A}-(1,1,1)_{B}, \quad(1,0,2)_{A}-(1,1,1)_{B}, \quad(1,1,1)_{A}-(2,0,1)_{B}$, and $(1,1,1)_{A}-(1,2,0)_{B}$ configurations, respectively.

Left and right tunneling 2 QSS are lines close to and parallel to the $(1,2,0)$ and $(0,2,1)$ to $(1,1,1)$ detuning boundaries, respectively. Working at these tunneling $2 \mathrm{QSS}$ require non-local gate times of the order $10-100 \mu \mathrm{s}$ in general, so the protection from tunneling noise has to be weighed against decoherence from long gate times. Linecuts along tunneling $2 \mathrm{QSS}$ are shown in Fig. $2 \mathrm{~d}$, g. At the intersection of left and right tunneling 2QSS lines, the conditions imposed by Makhlin invariants for non-local gates are not satisfied and Eq. (10) gives non-physical infinite gate time. It is therefore not possible to take advantage of the double tunneling $2 \mathrm{QSS}$. Equations of lines corresponding to tunneling 2QSS are

$$
\begin{aligned}
& \varepsilon_{\mathrm{mA}}=-\varepsilon_{\mathrm{A}}-2\left(U^{\prime}-U^{\prime \prime}\right), \quad\left(t_{\mathrm{IA}} 2 \mathrm{QSS}\right), \\
& \varepsilon_{\mathrm{mA}}=\varepsilon_{\mathrm{A}}-2\left(U^{\prime}-U^{\prime \prime}\right), \quad\left(t_{\mathrm{rA}} 2 \mathrm{QSS}\right), \\
& \varepsilon_{\mathrm{mB}}=\varepsilon_{\mathrm{B}}-2\left(U^{\prime}-U^{\prime \prime}\right), \quad\left(t_{\mathrm{IB}} 2 \mathrm{QSS}\right), \\
& \varepsilon_{\mathrm{mB}}=-\varepsilon_{\mathrm{B}}-2\left(U^{\prime}-U^{\prime \prime}\right) . \quad\left(t_{\mathrm{rB}} 2 \mathrm{QSS}\right) .
\end{aligned}
$$

The $\varepsilon_{\mathrm{mA} / \mathrm{B}}$ and $t_{\mathrm{IA} / \mathrm{B}}$ double 2QSS conditions for the qubits are given by the solution to the relevant pairs of simultaneous equations (Eqs. (15), (17) and Eqs. (16), (19)). There is no $\varepsilon 2 \mathrm{QSS}$ in the linear QD geometry. The full derivation of the $2 \mathrm{QSS}$ equations is given in Supplementary Note 6.

\section{Infidelity results}

Numerical simulations of Eq. (13) with noisy parameters are averaged to give infidelity $1-\langle\mathcal{F}\rangle$, and compared with that from analytics, $1-\left\langle\mathcal{F}_{\text {an }}\right\rangle$. Results are plotted in Fig. 3 for noise affecting the non-local gate for qubit $A$ only. The reason is that results for noisy qubit $B$ or both qubits noisy are qualitatively similar and the analytic fidelity acquires a simple expression, $\left\langle\mathcal{F}_{\text {an }}\right\rangle=\left\langle\mathcal{F}_{\text {an }}\right\rangle_{A} \times$ $\left\langle\mathcal{F}_{\text {an }}\right\rangle_{\mathrm{B}}$ (see Supplementary Discussion 2).

Infidelity results are shown in Fig. 3, which we refer to in the rest of this section. Numerical simulations are calculated by averaging over 500 noise realizations for every point in detuning space, except at double $t_{1}$ and $\varepsilon_{\mathrm{m}}$ 2QSS which are averaged over 100 realizations. The reason is the much longer gate times (up to $\sim 100 \mu \mathrm{s}$ ) at those points result in impractical runtimes. Infidelities from numerical simulations are shown in the leftmost column (Fig. 3a, e, i, m, q, u), analytical infidelity (Eq. (14)) in the second column (Fig. 3b, f, j, n, r, v). Each pair of infidelity color plots have the same scale to facilitate comparison.

Horizontal linecuts (circles) from numerical infidelity results are plotted with analytical infidelity (lines) in the third column (Fig. 3c, $\mathrm{g}, \mathrm{k}, \mathrm{o}, \mathrm{s}, \mathrm{w}$ ). Similarly, vertical linecuts (squares) are plotted in the rightmost column (Fig. $3 \mathrm{~d}, \mathrm{~h}, \mathrm{l}, \mathrm{p}, \mathrm{t}, \mathrm{x}$ ). Color scales for linecuts indicate the standard deviation of the noisy parameter at which the linecut was taken. All plots display good agreement between 

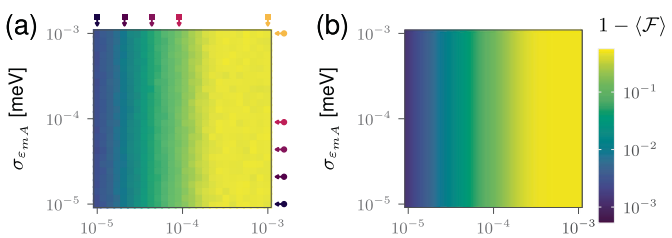

$\sigma_{\varepsilon_{A}}[\mathrm{meV}]$
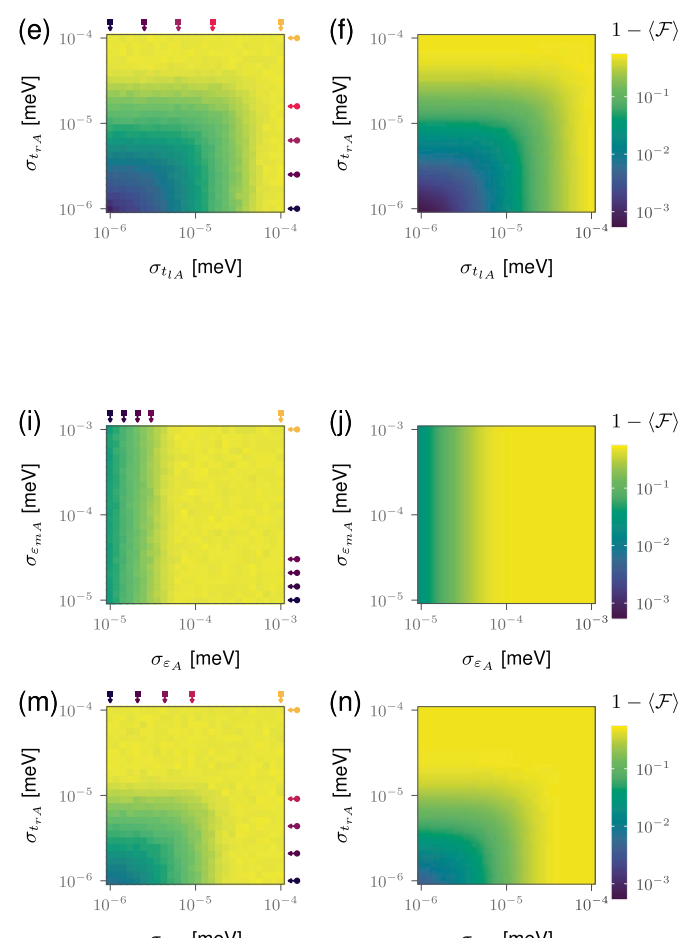

$\sigma_{t_{l A}}[\mathrm{meV}]$
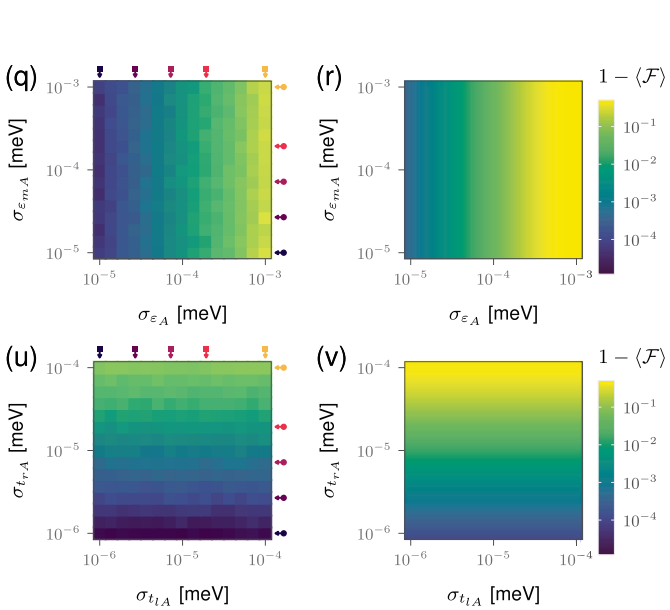

$\sigma_{t_{l A}}[\mathrm{meV}]$
$\mathrm{RX}, \varepsilon_{m}$ sweet spot
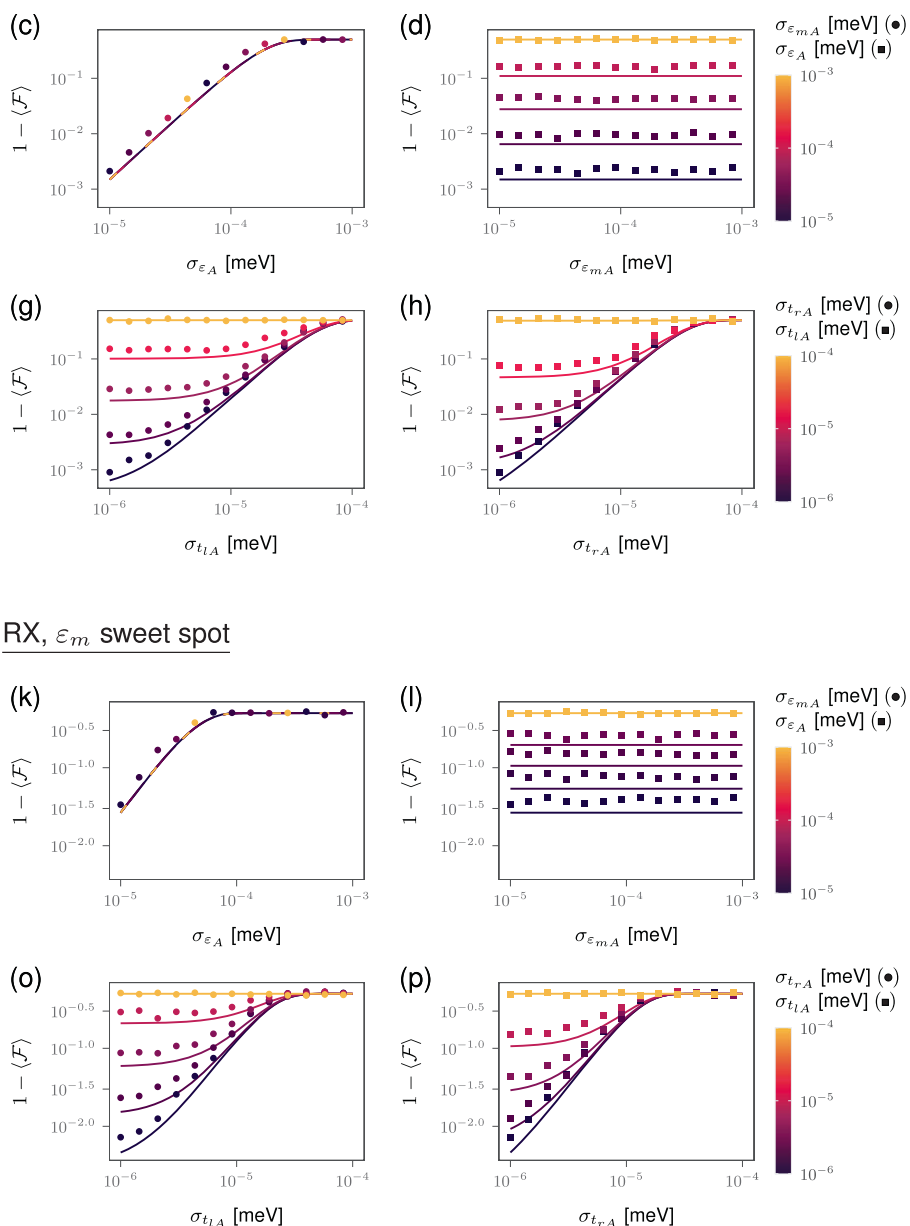

$\varepsilon_{m}$ and $t_{l}$ sweet spot
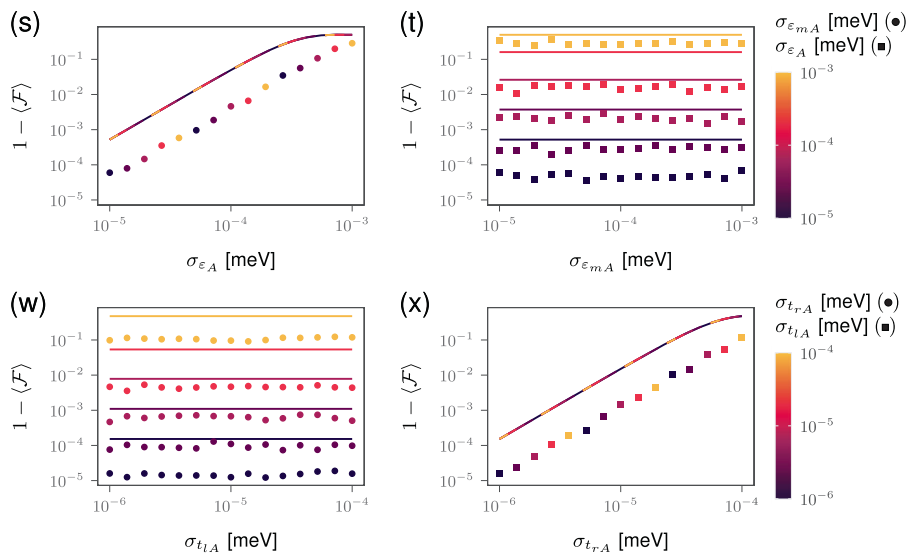

numerical and analytical calculations, except at $\varepsilon_{\mathrm{m}}$ and $t_{1}$ double 2QSS where $t_{0}$ increases significantly and the analytical formula over-predicts infidelity.

We analyze infidelity due to noise in detuning vs tunneling parameters separately because the contribution to infidelity depends on noise variance as well as the first derivative of the squares of charge admixtures. The latter yields terms that are smaller by a factor of $\sim t /(U+\varepsilon)$ for detuning derivatives compared to tunneling derivatives (see Supplementary Eqs. (134, 135),
Supplementary Note 5). When comparing infidelities from only detuning noise with that from only tunneling noise at the same working point, e.g., between panels $(a, b)$ and $(e, f)$, or between panels $(i, j)$ and $(m, n)$ of Fig. 3 , it is clear that infidelity is less sensitive to noise in detuning than tunneling.

Now, we examine the case when the RX and AEON qubits are operated at $\varepsilon_{\mathrm{m}} 2 \mathrm{QSS}$ and $\varepsilon 1 \mathrm{QSS}$. When $\arctan \left(t_{\mathrm{IA}} / t_{\mathrm{rA}}\right)=57^{\circ}$, the $\varepsilon_{\mathrm{mA}} 2 \mathrm{QSS}$ lies on $\varepsilon_{\mathrm{A}}=01 \mathrm{QSS}$. Vertical linecuts across $\sigma_{\varepsilon_{\mathrm{mA}}}$ (panels $\mathrm{d}$, I) show excellent agreement between analytical and 
Fig. 3 Gate infidelities under detuning or tunneling noise and 2QSS. $\mathbf{a}-\mathbf{h}$ Infidelity plots of AEON with qubit $A$ in the presence of only detuning noise (a-d) and only tunneling noise $(\mathbf{e}-\mathbf{h})$, while sitting at its single-qubit double sweet spot and the $\varepsilon_{m} 2 Q S S$. i-p Infidelity plots of $\mathrm{RX}(k=7$, for a comparable gate time with $\mathrm{AEON})$ with qubit $\mathrm{A}$ in the presence of only detuning noise (i-I) and only tunneling noise (m-p), while sitting at its single-qubit single sweet spot and the $\varepsilon_{\mathrm{m}} 2 \mathrm{QSS}$. q-x Infidelity plots with qubit $\mathrm{A}$ in the presence of only detuning noise (q-t) and only tunneling noise (u-x), while sitting at the $\varepsilon_{\mathrm{m}}$ and $t_{1} 2 \mathrm{QSS}$. Panels $(\mathbf{a}, \mathbf{e}, \mathbf{i}, \mathbf{m}, \mathbf{q}, \mathbf{u})$ are numerical simulations (Eq. (13)) averaged over 500 $(\mathbf{a}, \mathbf{e}, \mathbf{i}, \mathbf{m})$ and $100(\mathbf{q}, \mathbf{u})$ noise realizations. Panels $(\mathbf{b}, \mathbf{f}, \mathbf{j}, \mathbf{n}, \mathbf{r}, \mathbf{v})$ are analytical calculations (Eq. (14)) and agree well with numerical simulations, as evident from comparisons with linecuts. Corresponding analytical and numerical plots share the same color scale. Panels (c, $\mathbf{k}$, s) show horizontal linecuts (circles), and panels (d, $\mathbf{I}, \mathbf{t})$ show vertical linecuts (squares) from the numerical result in panels (a, $\mathbf{i}, \mathbf{q})$, and agree very well with analytical calculations (lines) at the $\varepsilon_{\mathrm{m}}$ 2QSS, while analytical infidelity overestimates by an order of magnitude for the $\varepsilon_{\mathrm{m}}$ and $t_{1}$ double 2QSS. Panels $(\mathbf{d}, \mathbf{I}, \mathbf{t})$ show that infidelity is independent of middle detuning noise $\sigma_{\varepsilon_{\mathrm{mA}}}$ which confirms the $\varepsilon_{\mathrm{mA}} 2 \mathrm{QSS}$. Panel (w) shows that infidelity is also independent of the left tunneling noise $\sigma_{t_{\mathrm{IA}}}$ which confirms that it is the double $2 \mathrm{QSS}$ of $\varepsilon_{\mathrm{mA}}$ and $t_{\mathrm{IA}}$. In contrast, infidelity increases with noise detuning $\sigma_{\varepsilon_{A}}$ (panels $\mathbf{c}, \mathbf{k}, \mathbf{s}$ ) because there is no such 2QSS, and in right tunneling $\sigma_{t_{\mathrm{rA}}}$ when not operated at the $t_{\mathrm{rA}} 2 \mathrm{QSS}$ (panels $\mathbf{h}, \mathbf{p}, \mathbf{x}$ ). Color scales in rightmost column represent the numerical value of noise amplitude at which the linecuts are taken. Results for noisy qubit B or both qubits noisy are qualitatively similar (see Supplementary Discussion 2).
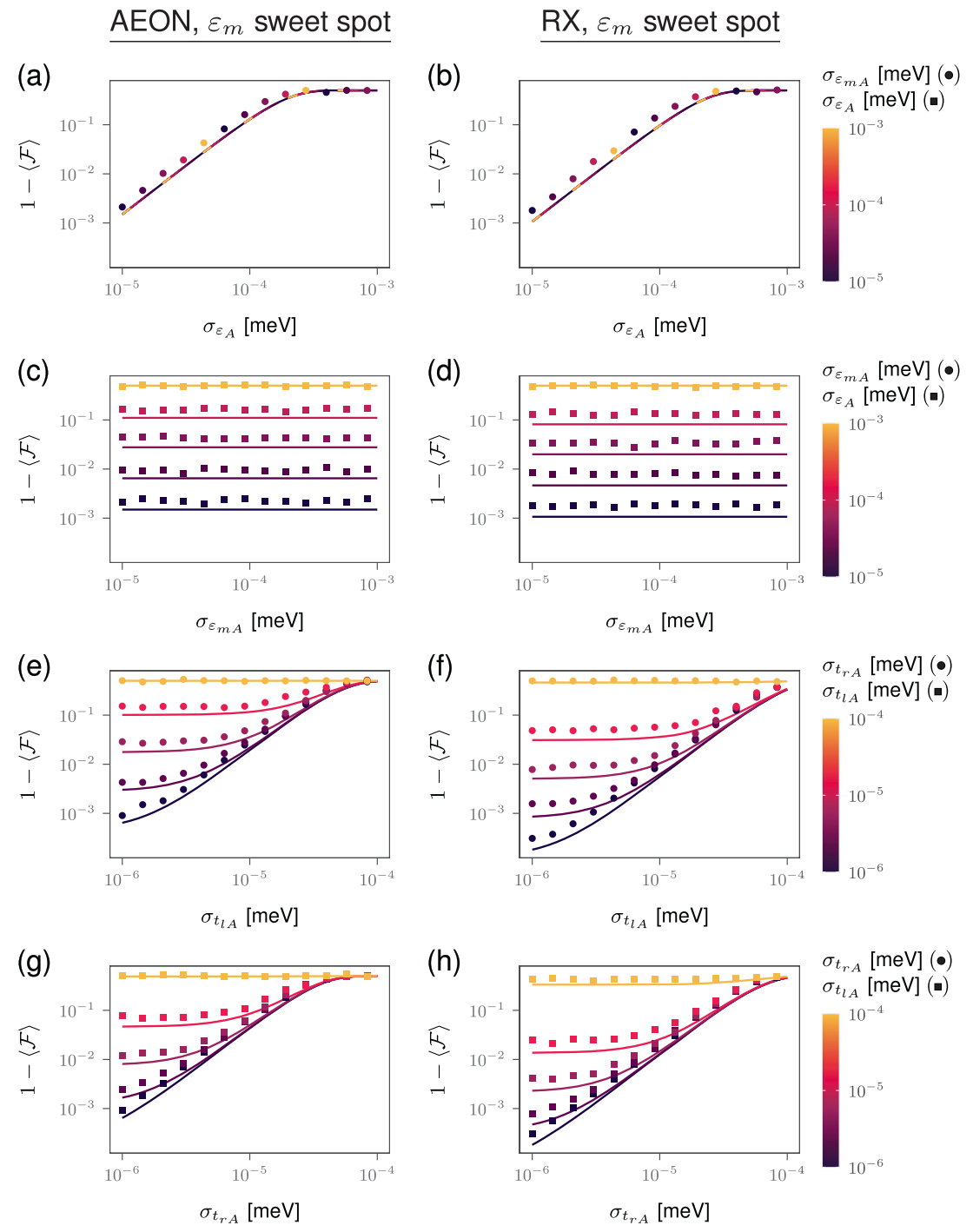

Fig. 4 Comparison of infidelities for the fastest gate times $(k=1)$ for the AEON $\left(t_{0}=450 \mathrm{~ns}\right)$ and RX ( $\left.t_{0}=64 \mathrm{~ns}\right)$, when the $\varepsilon_{\mathrm{mA}} 2 \mathrm{QSS}$ overlaps with the $\boldsymbol{\varepsilon}_{\mathbf{A}}=\mathbf{0}$ line. Left column (panels $\left.\mathbf{a}, \mathbf{c}, \mathbf{e}, \mathbf{g}\right)$ are identical with panels $(\mathbf{c}, \mathbf{d}, \mathbf{g}, \mathbf{h})$ of Fig. 3. Right column (panels $\left.\mathbf{b}, \mathbf{d}, \mathbf{f}, \mathbf{h}\right)$ are similar to panels $(\mathbf{k}, \mathbf{l}, \mathbf{0}, \mathbf{p})$ of Fig. 3 , except that they are calculated for $k=1$. Comparing each row, it is clear that the RX performs slightly better than the AEON, as expected for a faster qubit.

numerical calculations. In order to fairly compare RX and AEON infidelities at these points, we chose $k$ (Eq. (10)) such that they have comparable gate times of $450 \mathrm{~ns}$ ( $k=1$, AEON) and $448 \mathrm{~ns}$ $(k=7, \mathrm{RX})$. For comparable gate times, it is favorable to work at the AEON 1QSS, suggesting that while the RX operates faster due to a greater dipole-dipole interaction, it is more susceptible to charge noise. However, with the fastest gate time $(k=1)$ of $64 \mathrm{~ns}$ for RX, a slightly better fidelity (Fig. 4b, d, f, h) is achieved, demonstrating the natural relationship between fast gates and improved fidelity. 
Next, when the $\varepsilon_{\mathrm{mA}} 2 \mathrm{QSS}$ line is slanted, e.g., for $\arctan \left(t_{\mathrm{IA}} / t_{\mathrm{rA}}\right)=45^{\circ}$, it intersects the $t_{\mathrm{IA}} 2 \mathrm{QSS}$. At this double $2 \mathrm{QSS}$, where gate time is longer than when operating on $\mathrm{RX}$ or AEON 1QSS, vertical linecuts along $\sigma_{\varepsilon_{\mathrm{mA}}}$ (Fig. 3t) and horizontal linecuts along $\sigma_{t_{\mid A}}$ (Fig. $3 \mathrm{w}$ ) display constant infidelities, demonstrating the double 2 QSS character of the working point. However, the analytical formula over-predicts infidelity compared with numerical simulations, as shown in Fig. 3s, t, w, x.

Finally, there is no $\varepsilon_{A} 2 Q S S$. As expected, linecuts (Fig. $3 c, g, h, k$, $\circ, \mathrm{p})$ show rising infidelity with greater $\sigma_{\varepsilon_{\mathrm{A}}}$, and excellent analytical and numerical agreement.

\section{DISCUSSION}

Here, we describe optimal choices of 2QSS working points, all of which fall on the $\varepsilon_{\mathrm{m}}$ 2QSS. First, if it is desired that qubits are protected from noise during both single- and two-qubit operation with minimal experimental control, then it is best to work on the intersection of $\varepsilon_{\mathrm{m}} 2 \mathrm{QSS}$ and $\varepsilon 1 \mathrm{QSS}$. This requires tunnel coupling ratios to be tuned to $\arctan \left(t_{\mathrm{IA}} / t_{\mathrm{rA}}\right)=57^{\circ}$ during non-local operations. At this ratio, the $\varepsilon_{\mathrm{mA}} 2 \mathrm{QSS}$ is a vertical line passing through $A E O N$ and $R X \& 1 Q S S$. During single-qubit gates, in order to return to the RX $1 \mathrm{QSS}$, tunnel couplings must be re-equalized. The AEON does not require retuning since its $1 \mathrm{QSS}$ is independent of tunneling.

Further optimization for RX can be done. Because tunneling asymmetry shifts its $\varepsilon 1 \mathrm{QSS}$, this can be made to coincide with the $\varepsilon_{\mathrm{m}}$ 2QSS for one particular tunneling ratio, $\arctan \left(t_{1} / t_{\mathrm{r}}\right)=37.7^{\circ}$ for the parameters used. This is presented in Fig. 5, where panel (a) plots 1 QSS and 2QSS against tunneling ratio, and panel (b) illustrates where they coincide on a color plot of non-local gate time. Left of the midline, gate time is longer, $t_{0}=198 \mathrm{~ns}$.

Next, even when these advantages cannot be exploited due to say, limited tunability of tunnel coupling, the intersection of $\varepsilon_{\mathrm{m}}$ 2QSS line with one of the tunneling 2QSS provides another avenue for fidelity improvement. This intersection between $\varepsilon_{\mathrm{m}}$ and $t_{1}$ 2QSS is shown by the white circle at the lower left of Fig. $2 b$, e. Comparing infidelity plots in Fig. 3, the best fidelities are found when working at the $\varepsilon_{\mathrm{m}}$ and $t_{\mathrm{l}}$ double 2QSS. The experimental complication to working at this point is the movement to and from the 1QSS, during single-qubit gates.

The conditions for the existence of both $t_{1}$ and $t_{r} 2 \mathrm{QSS}$ are equivalent to the requirement for the qubit to remain in the $(1,1,1)$ region, $-\left(U-U^{\prime}\right)<\left(\varepsilon_{\mathrm{mA}}+U^{\prime}-U^{\prime \prime}\right) \pm \varepsilon_{\mathrm{A}}<U-U^{\prime}$. The conditions
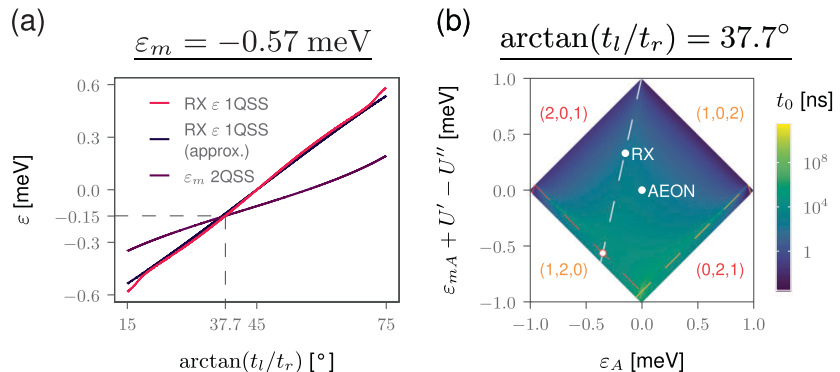

Fig. 5 RX single-qubit sweet spot (1QSS) and two-qubit sweet spot (2QSS) dependence on tunneling ratio. a The red curve shows the exact dependence of RX $\varepsilon 1 \mathrm{QSS}$ on $\arctan \left(t_{1} / t_{\mathrm{r}}\right)$. The blue line, from ref. ${ }^{36}$, shows the approximate dependence, $\varepsilon \approx-(8 \Delta / 5) y$, where $\Delta=U-2 U^{\prime}+U^{\prime \prime}-\varepsilon_{m}$ and small tunneling asymmetry $y=\sin \left(\pi / 4-\arctan \left(t_{1} / t_{\mathrm{r}}\right)\right)$. The purple curve shows the $\varepsilon_{\mathrm{m}} 2 \mathrm{QSS}$ position as a function of $\arctan \left(t_{1} / t_{r}\right)$. At the intersection where both $1 \mathrm{QSS}$ and 2QSS share the same tunneling ratio, $\varepsilon=-0.15 \mathrm{meV}$, $\arctan \left(t_{1} / t_{\mathrm{r}}\right)=37.7^{\circ}$, for parameters used in this study. $\mathbf{b}$ The gate times at the tunneling ratio $\arctan \left(t_{1} / t_{r}\right)=37.7^{\circ}$. At the intersection of $\varepsilon_{\mathrm{m}}$ 2QSS (white dashed line) and RX 1QSS, non-local gate time is $t_{0}=198 \mathrm{~ns}$. for $\varepsilon_{\mathrm{mA}}$ and $\varepsilon_{\mathrm{mB}}$ 2QSS to exist are $\operatorname{sgn}\left(C_{51}-C_{11}\right)=\operatorname{sgn}\left(C_{21}-C_{11}\right)$ and $\operatorname{sgn}\left(C_{13}-C_{11}\right)=\operatorname{sgn}\left(C_{14}-C_{11}\right)$, respectively. These conditions are automatically satisfied in a linear QD array. The full derivation is given in Supplementary Note 6.

Having studied fidelities of specific working points on 2QSS, it is reasonable to ask if a global fidelity optimum might exist. In Fig. 6 , when there is only detuning noise in qubit $A$ (panels $a, c$, d), the global fidelity optimum is a single point lying on $\varepsilon_{\mathrm{mA}}$ $2 \mathrm{QSS}$, in the lower half of the $(1,1,1)$ charge region. Analytical calculations show infidelity $\approx 10^{-10}$; numerical calculations give infidelity $\approx 10^{-6}$. Both meet fault-tolerance thresholds, $1-\mathcal{F}<10^{-457,58}$ and $10^{-659}$. This is significant because when tunneling noise is negligible, working at this global optimum will achieve fault-tolerance.

However, when there is only tunneling noise, the global fidelity optimum is located in the upper right quadrant of the $(1,1,1)$ region (panels $b, e)$, and infidelity in the lower half of the $(1,1,1)$ region increases significantly. Because we expect both detuning and tunneling noise to affect EO qubits, and infidelity is approximately additive, the optimum point for global fidelity from detuning noise may be limited by infidelity from tunneling noise.

Above, we analyzed results when qubit $A$ is noisy. Similar results apply when qubit B or both qubits are noisy. We also assumed noiseless single-qubit gates. Next, we discuss fidelity optimization with noisy single-qubit gates.

CPHASE involves an additional $z$-rotation for each qubit. At simultaneous $1 \mathrm{QSS}$ and 2QSS, $z$-rotations times are $t_{1}=t_{2} \approx 1 \mathrm{~ns}$ (AEON) and $0.4 \mathrm{~ns}(\mathrm{RX})$ for $\hbar \omega_{\mathrm{A} / \mathrm{B}} \approx 1 \mathrm{meV}$. Both single-qubit $z$-gates are 2 orders of magnitude faster than the non-local gate. Therefore, at these sweet spot intersections, it is likely that the non-local gate limits fidelity.

As discussed, the global minimum need not lie on SS intersections, and is dependent on the dominant noise parameter. This necessitates a complete understanding of the noise power of each parameter. In addition, noise may be correlated. However, noise spectroscopy may not be trivial to implement since noise acts on multiple axes in these qubits, although theoretical progress in multiaxis noise spectroscopy had been made ${ }^{53,60}$. Fortunately, the same parameter space governs single and two-qubit gates; perhaps a simple formula might relate single and two-qubit fidelities.

CNOT requires single-qubit $x$ - and $z$-rotations. Because pulse gating for AEON rotates the qubit around the $-(x+z)$ axis, singlequbit rotations might benefit from optimal control pulses ${ }^{61}$. On the other hand, RX uses microwave control and can directly perform $x$-rotations, the speed of which depends on drive amplitude. Because AEON and RX can transform into each other, they should be able to take advantage of the features each one offers for further optimization.

In summary, we studied capacitive two-qubit CPHASE and CNOT gates for EO qubits with a focus on AEON and RX proposals. We demonstrated the existence of $\varepsilon_{\mathrm{m}}, t_{1}$, and $t_{\mathrm{r}}$ 2QSS for each qubit, and provided conditions for their existence. We showed how the $\varepsilon_{\mathrm{m}}$ 2QSS can be tuned to intersect with $\varepsilon 1 \mathrm{QSS}$, requiring only tuning of tunnel coupling ratios. This has the benefit of operating the qubit at both $1 \mathrm{QSS}$ and 2QSS. We also showed that double $2 \mathrm{QSS}$ also exist- $\varepsilon_{\mathrm{mA}}$ with $t_{\mathrm{IA}}$ (qubit A) or $\varepsilon_{\mathrm{mB}}$ with $t_{\mathrm{rB}}$ (qubit B)—providing another avenue for fidelity improvements. Importantly, the global fidelity optimum lies along the $\varepsilon_{m} 2 \mathrm{QSS}$, with a fidelity better than the fault-tolerance threshold when tunneling noise is negligible.

Our infidelity results illustrate the stringent requirement for qubits. Best fidelities are obtained when working at the double $\varepsilon_{\mathrm{mA}}$ and $t_{\mathrm{IA}} 2 \mathrm{QSS}$. However, only with extremely low noise, e.g., $\sigma_{\varepsilon_{\mathrm{A}}}<10^{-5} \mathrm{meV}$ or $\sigma_{t_{\mathrm{rA}}}<10^{-5} \mathrm{meV}$, can the fault-tolerance conditions be met. The fidelities in our study were computed for noisy non-local gate and noiseless single-qubit gates. In reality, because 
$\arctan \left(t_{l A} / t_{r A}\right)=45^{\circ}$
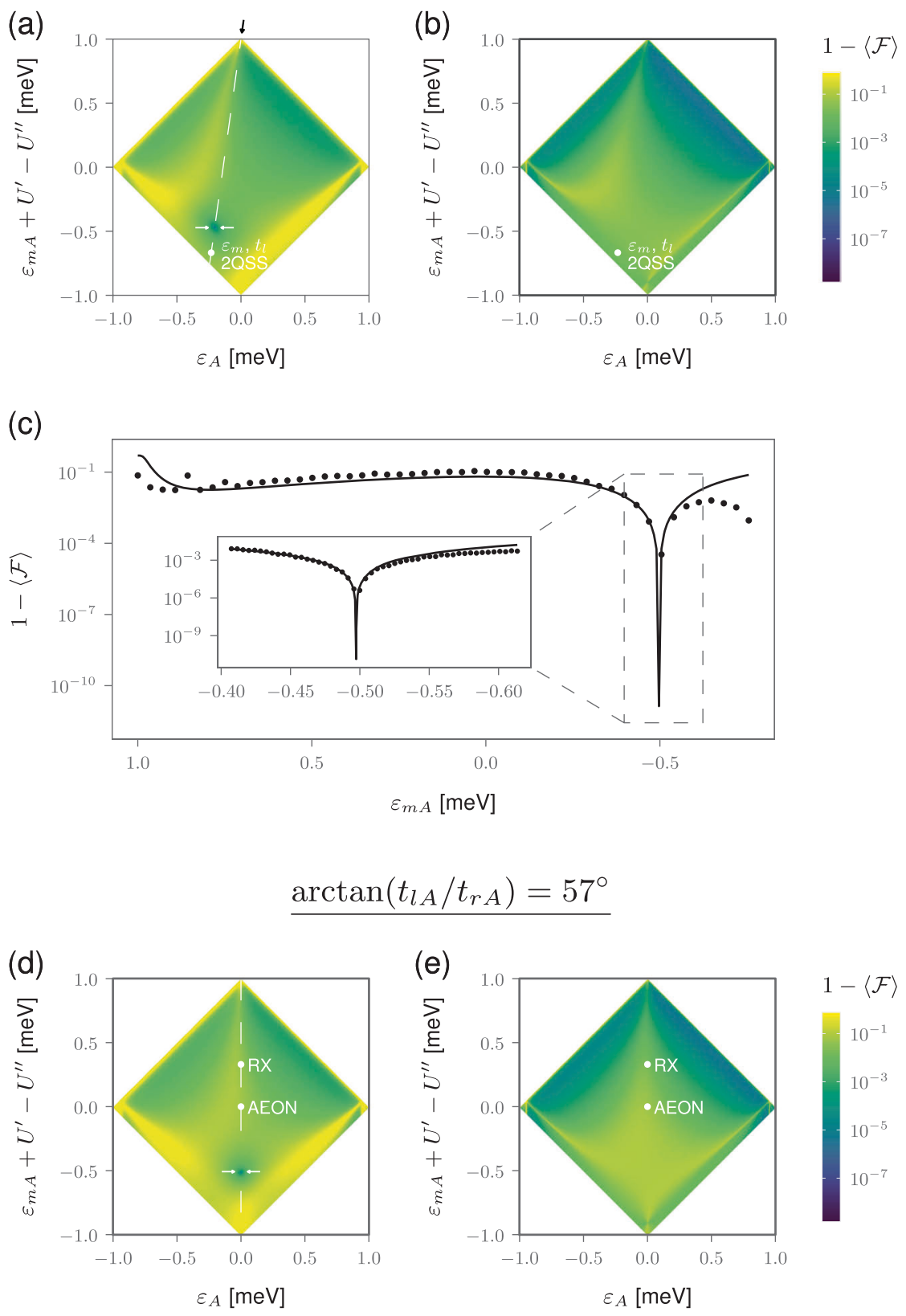

Fig. 6 Color plots of analytical formula (Eq. (14)) of infidelity in detuning parameter space with fixed standard deviation of noise. There is only detuning noise, $\sigma_{\varepsilon_{\mathrm{mA}}}=\sigma_{\varepsilon_{\mathrm{A}}}=10^{-4} \mathrm{meV}$ for $(\mathbf{a}, \mathbf{c}, \mathbf{d})$, and only tunneling noise, $\sigma_{t_{\mid \mathrm{A}}}=\sigma_{t_{\mathrm{rA}}}=10^{-5} \mathrm{meV}$ for (b, e). Tunneling parameters are $t_{\mathrm{IA}}=t_{\mathrm{rA}}$ in panels $(\mathbf{a}, \mathbf{b}, \mathbf{c})$, and $\arctan \left(t_{\mathrm{IA}} / t_{\mathrm{rA}}\right)=57^{\circ}$ in panels $(\mathbf{d}, \mathbf{e})$. a The global optimum when there is only detuning noise lies along the $\varepsilon_{\mathrm{m}}$ $2 \mathrm{QSS}$. This global optimum is in a region of large $\varepsilon_{\mathrm{m}}$ for tunneling noise. The global optimum lies at a point which has extremely small gate times that require timing precision of $\sim$ ps or better, which may be currently out of reach experimentally. $\mathbf{b}$ When there is only tunneling noise, the global optimum lies in the region near the upper right boundary of the $(1,1,1)$ region. The optimal point in panel $(\mathbf{a})$ is now a point with significantly larger infidelity $\left(1-\left\langle\mathcal{F}_{\text {an }}\right\rangle \approx 10^{-2}\right)$. c Infidelity linecut along the $\varepsilon_{\mathrm{m}} 2 \mathrm{QSS}$. The analytical (line) and numerically simulated (points) infidelities agree well, although they start to deviate past the global optimum. Global infidelity optimum is better than $10^{-10}$ from analytical calculations, and $10^{-6}$ from numerical simulations. $\mathbf{d}$ With only detuning noise, and tuning tunneling parameters so that the $\varepsilon_{\mathrm{m}} 2 \mathrm{QSS}$ is along $\varepsilon=0$, the global optimum still lies on the $\varepsilon_{\mathrm{m}}$ 2QSS. However, the gate times become very large near bottom corner of the $(1,1,1)$ region $($ see Fig. 2) and becomes impractical to implement. e With only tunneling noise and the $\varepsilon_{\mathrm{m}}$ 2QSS is along $\varepsilon=0$, the infidelity again rises significantly at the region where it was the global optimum in panel (d) when there was only detuning noise. In reality, there should be noise in both detuning and tunneling, and infidelities are approximately additive, demonstrating the difficulty of finding a truly global optimal working point.

both qubits will be noisy and single-qubit gates will similarly be afflicted, the requirements are likely to be even stricter.

Given recent experimental progress in scaling up of QD arrays and capacitive coupling, our results should contribute toward the realization of high fidelity two-qubit gates.

\section{METHODS}

\section{Numerical simulations}

We numerically calculate the average fidelity (Eq. (13)) of the non-local two-qubit gate by averaging over 500 different simulations of noise for each noisy parameter $\tilde{n}_{i}$, except at double $t_{l}$ and $\varepsilon_{\mathrm{m}} 2 \mathrm{QSS}$ points which 
are averaged over 100 realizations. Each noisy time series $\delta n_{i}(t)$ is simulated with the desired spectrum of Eq. (8) from the algorithm of refs. ${ }^{47,48}$, which generates for every positive $\omega_{k}$ value, two Gaussian distributed random numbers to represent the real and imaginary parts of the spectrum. After scaling by $\sqrt{S\left(\omega_{k}\right) / 2}$, an inverse FFT produces the desired noisy time series. Our modification consists of shifting the mean of the generated time series to zero and then rescaling its variance to the desired value (see Supplementary Method 4). At each time step, the charge admixtures $\alpha_{i}, \beta_{i}$, and the interaction terms $\tilde{V}_{i}$ are computed, and the full unitary evolution with the exact gate sequence is calculated. All simulations in Figs. 3 and 4 are performed with cutoff frequencies, $\omega_{1} /$ $2 \pi=66.7 \mathrm{kHz}, \omega_{\mathrm{h}} / 2 \pi=50 \mathrm{GHz}$.

\section{Triple quantum dot potential and parameters}

The Hubbard model describes both intra-TQD and inter-TQD interactions. Intra-TQD interactions comprise QD detunings, tunnel couplings as well as intra and inter-dot Coulomb energies. Inter-TQD interaction comprise inter-dot Coulomb energies only, when tunnel coupling between the TQDs (QD 3 and 4) is zero. Parameters of inter-TQD interactions are calculated from a model confinement potential and intra-TQD parameters from estimates in literature. See Supplementary Note 7 for a discussion of this approach.

The TQD potential is modeled as a 2D tri-quadratic potential, $v_{\text {pot }}(\boldsymbol{r})=\min \left[v_{\text {pot, } 1}(\boldsymbol{r}), v_{\text {pot, } 2}(\boldsymbol{r}), v_{\text {pot }, 3}(\boldsymbol{r})\right]$, where the $i$-th QD well at position $\boldsymbol{R}_{i}$ is $v_{\text {pot, } \mathrm{i}}(\boldsymbol{r})=\frac{1}{2} m \omega_{0}^{2}\left(\left|\boldsymbol{x}-\boldsymbol{R}_{i}\right|^{2}+\boldsymbol{y}^{2}\right)+\varepsilon_{i}$, whose eigenfunctions are the $2 \mathrm{D}$ Fock-Darwin wavefunctions ${ }^{62}$. The $2 \mathrm{D}$ character of the potential is a good approximation to electrostatically gated QDs, given the tight confinement in the $z$-direction. The confinement $m \omega_{0}=\hbar / a_{\mathrm{B}}^{2}$, where $a_{\mathrm{B}}$ is the Bohr radius. Treating neighboring potential wells as perturbations, dot-centered, normalized single-electron wavefunctions $\psi_{i}$ of the TQD potential are constructed from the Fock-Darwin wavefunctions using the method of Löwdin Orthogonalization ${ }^{63,64}$, from which the three-electron wavefunctions are formulated. Numerical values take reference from refs. ${ }^{20,39}: a=50 \mathrm{~nm}, a_{\mathrm{B}}=$ $25 \mathrm{~nm} ; R=160 \mathrm{~nm} ; U_{i}=U=2.8 \mathrm{meV} ; U_{12}=U_{23}=U_{45}=U_{56}=U^{\prime}=1.8$ $\mathrm{meV} ; U_{13}=U_{46}=U^{\prime \prime}=0.9 \mathrm{meV}$. For both AEON and RX, $t_{\mathrm{IA}}=t_{\mathrm{BB}}=0.12 \mathrm{meV}$, $\varepsilon_{\mathrm{A}}=\varepsilon_{\mathrm{B}}=0$. For AEON, $\varepsilon_{\mathrm{mA}}=\varepsilon_{\mathrm{mB}}=-0.9 \mathrm{meV}$. For $\mathrm{RX}, \varepsilon_{\mathrm{mA}}=\varepsilon_{\mathrm{mB}}=-0.57 \mathrm{meV}$. Direct Coulomb integrals in the capacitive interaction are $\mathcal{V}_{i j}=\left(q^{2} / 4 \pi \epsilon_{r} \epsilon_{0}\right) \iint\left|\psi_{i}\left(\boldsymbol{r}_{1}\right)\right|^{2} \frac{1}{\left|\boldsymbol{r}_{1}-\boldsymbol{r}_{2}\right|}\left|\psi_{j}\left(\boldsymbol{r}_{2}\right)\right|^{2} d \boldsymbol{r}_{1} d \boldsymbol{r}_{2}$, where we take silicon relative permittivity, $\epsilon_{\mathrm{r}}=11.68$. Even though the exact form of $\mathrm{QD}$ confinement potential depends on the device, an advantage of modeling the TQD potential as tri-quadratic is that each integral is analytically tractable. With these parameters, a check shows that direct Coulomb integrals are a factor of $10^{4}$ greater than the spin-dependent exchange Coulomb integrals (see Supplementary Discussion 3), validating our assumption of capacitive non-local gating.

\section{DATA AVAILABILITY}

The data that support the findings of this study are available at https://doi.org/ 10.21979/N9/TYUUVS.

\section{CODE AVAILABILITY}

The computer code used in generating the data are available from the corresponding author on reasonable request.

Received: 29 March 2021; Accepted: 23 June 2021; Published online: 16 July 2021

\section{REFERENCES}

1. Loss, D. \& DiVincenzo, D. P. Quantum computation with quantum dots. Phys. Rev. A 57, 120-126 (1998).

2. Zwanenburg, F. A. et al. Silicon quantum electronics. Rev. Mod. Phys. 85, 961-1019 (2013).

3. Yoneda, J. et al. A quantum-dot spin qubit with coherence limited by charge noise and fidelity higher than 99.9\%. Nat. Nanotechnol. 13, 102-106 (2018).

4. Zajac, D. M. et al. Resonantly driven CNOT gate for electron spins. Science 359, 439-442 (2018).
5. Foletti, S., Bluhm, H., Mahalu, D., Umansky, V. \& Yacoby, A. Universal quantum control of two-electron spin quantum bits using dynamic nuclear polarization. Nat. Phys. 5, 903-908 (2009).

6. Bluhm, H. et al. Dephasing time of GaAs electron-spin qubits coupled to a nuclear bath exceeding $200 \mu$ s. Nat. Phys. 7, 109-113 (2011).

7. Kim, D. et al. Quantum control and process tomography of a semiconductor quantum dot hybrid qubit. Nature 511, 70-74 (2014).

8. Kim, D. et al. High-fidelity resonant gating of a silicon-based quantum dot hybrid qubit. npj Quantum Inf. 1, 15004 (2015).

9. Thorgrimsson, B. et al. Extending the coherence of a quantum dot hybrid qubit. npj Quantum Inf. 3, 32 (2017).

10. Cerfontaine, P. et al. Closed-loop control of a GaAs-based singlet-triplet spin qubit with $99.5 \%$ gate fidelity and low leakage. Nat. Commun. 11, 4144 (2020).

11. Schröer, D. et al. Electrostatically defined serial triple quantum dot charged with few electrons. Phys. Rev. B 76, 075306 (2007).

12. Laird, E. A. et al. Coherent spin manipulation in an exchange-only qubit. Phys. Rev. B 82, 075403 (2010).

13. Gaudreau, L. et al. Coherent control of three-spin states in a triple quantum dot. Nat. Phys. 8, 54-58 (2012).

14. DiVincenzo, D. P., Bacon, D., Kempe, J., Burkard, G. \& Whaley, K. B. Universal quantum computation with the exchange interaction. Nature 408, 339-342 (2000).

15. Shi, Z. et al. Fast hybrid silicon double-quantum-dot qubit. Phys. Rev. Lett. 108, 140503 (2012).

16. Koh, T. S., Gamble, J. K., Friesen, M., Eriksson, M. A. \& Coppersmith, S. N. Pulsegated quantum-dot hybrid qubit. Phys. Rev. Lett. 109, 250503 (2012).

17. Russ, M. \& Burkard, G. Three-electron spin qubits. J. Phys. Condens. Matter 29, 393001 (2017)

18. Srinivasa, V. \& Taylor, J. M. Capacitively coupled singlet-triplet qubits in the double charge resonant regime. Phys. Rev. B 92, 235301 (2015).

19. Calderon-Vargas, F. A. \& Kestner, J. P. Directly accessible entangling gates for capacitively coupled singlet-triplet qubits. Phys. Rev. B 91, 035301 (2015).

20. Neyens, S. F. et al. Measurements of capacitive coupling within a quadruplequantum-dot array. Phys. Rev. Appl. 12, 064049 (2019).

21. MacQuarrie, E. R. et al. Progress toward a capacitively mediated CNOT between two charge qubits in Si/SiGe. npj Quantum Inf. 6, 81 (2020).

22. Fujita, T., Baart, T. A., Reichl, C., Wegscheider, W. \& Vandersypen, L. M. Coherent shuttle of electron-spin states. npj Quantum Inf. 3, 22 (2017).

23. Feng, M., Kwong, C. J., Koh, T. S. \& Kwek, L. C. Coherent transfer of singlettriplet qubit states in an architecture of triple quantum dots. Phys. Rev. B 97, 245428 (2018).

24. Mills, A. R. et al. Shuttling a single charge across a one-dimensional array of silicon quantum dots. Nat. Commun. 10, 1063 (2019).

25. Yoneda, J. et al. Coherent spin qubit transport in silicon. Nat. Commun. 12, 4114 (2021).

26. Ginzel, F., Mills, A. R., Petta, J. R. \& Burkard, G. Spin shuttling in a silicon double quantum dot. Phys. Rev. B 102, 195418 (2020).

27. $\mathrm{Mi}, \mathrm{X}$. et al. Circuit quantum electrodynamics architecture for gate-defined quantum dots in silicon. App. Phys. Lett. 110, 43502 (2017).

28. Stockklauser, A. et al. Strong coupling cavity QED with gate-defined double quantum dots enabled by a high impedance resonator. Phys. Rev. X 7, 11030 (2017).

29. Fong, B. H. \& Wandzura, S. M. Universal quantum computation and leakage reduction in the 3-qubit decoherence free subsystem. Quantum Info. Comput. 11, 1003-1018 (2011).

30. Doherty, A. C. \& Wardrop, M. P. Two-qubit gates for resonant exchange qubits. Phys. Rev. Lett. 111, 050503 (2013).

31. Shim, Y. P. \& Tahan, C. Charge-noise-insensitive gate operations for always-on, exchange-only qubits. Phys. Rev. B 93, 121410(R) (2016).

32. Paladino, E., Galperin, Y., Falci, G. \& Altshuler, B. L. 1/f noise: Implications for solidstate quantum information. Rev. Mod. Phys. 86, 361-418 (2014).

33. Fei, J. et al. Characterizing gate operations near the sweet spot of an exchangeonly qubit. Phys. Rev. B 91, 205434 (2015).

34. Frees, A., Mehl, S., Gamble, J. K., Friesen, M. \& Coppersmith, S. N. Adiabatic twoqubit gates in capacitively coupled quantum dot hybrid qubits. npj Quantum Inf. 5, 73 (2019).

35. Medford, J. et al. Quantum-dot-based resonant exchange qubit. Phys. Rev. Lett. 111, 050501 (2013).

36. Taylor, J. M., Srinivasa, V. \& Medford, J. Electrically protected resonant exchange qubits in triple quantum dots. Phys. Rev. Lett. 111, 050502 (2013).

37. Russ, M. \& Burkard, G. Asymmetric resonant exchange qubit under the influence of electrical noise. Phys. Rev. B 91, 235411 (2015).

38. Wardrop, M. P. \& Doherty, A. C. Characterization of an exchange-based two-qubit gate for resonant exchange qubits. Phys. Rev. B 93, 075436 (2016). 
39. Das Sarma, S., Wang, X. \& Yang, S. Hubbard model description of silicon spin qubits: charge stability diagram and tunnel coupling in Si double quantum dots. Phys. Rev. B 83, 235314 (2011).

40. Schrieffer, J. R. \& Wolff, P. A. Relation between the Anderson and Kondo Hamiltonians. Phys. Rev. 149, 491-492 (1966).

41. Gros, C., Joynt, R. \& Rice, T. M. Antiferromagnetic correlations in almost-localized Fermi liquids. Phys. Rev. B 36, 381-393 (1987).

42. MacDonald, A. H., Girvin, S. M. \& Yoshioka, D. t/U expansion for the Hubbard model. Phys. Rev. B 37, 9753-9756 (1988).

43. Chan, K. W. et al. Assessment of a silicon quantum dot spin qubit environment via noise spectroscopy. Phys. Rev. Appl. 10, 44017 (2018).

44. Struck, T. et al. Low-frequency spin qubit energy splitting noise in highly purified ${ }^{28} \mathrm{Si} / \mathrm{SiGe}$. npj Quantum Inf. 6, 1-7 (2020).

45. Russ, M., Ginzel, F. \& Burkard, G. Coupling of three-spin qubits to their electric environment. Phys. Rev. B 94, 165411 (2016).

46. Huang, P., Zimmerman, N. M. \& Bryant, G. W. Spin decoherence in a two-qubit CPHASE gate: the critical role of tunneling noise. npj Quantum Inf. 4, 62 (2018).

47. Timmer, J. \& Koenig, M. On generating power law noise. Astron. Astrophys. 300, 707-707 (1995).

48. Patzelt, F. Python package to generate gaussian (1/f)**beta noise. https://github. com/felixpatzelt/colorednoise (2019).

49. Makhlin, Y. Nonlocal properties of two-qubit gates and mixed states and optimization of quantum computations. Quant. Inf. Proc. 1, 243-252 (2004).

50. Pal, A., Rashba, E. I. \& Halperin, B. I. Exact CNOT gates with a single nonlocal rotation for quantum-dot qubits. Phys. Rev. B 92, 125409 (2015).

51. Łuczak, J. \& Bułka, B. R. Two-qubit logical operations in three quantum dots system. J. Phys. Condens. Matter 30, 225601 (2018).

52. Wang, X., Yu, C. S. \& Yi, X. X. An alternative quantum fidelity for mixed states of qudits. Phys. Lett. A 373, 58-60 (2008).

53. Green, T. J., Sastrawan, J., Uys, H. \& Biercuk, M. J. Arbitrary quantum control of qubits in the presence of universal noise. New J. Phys. 15, 095004 (2013).

54. Nielsen, M. A. \& Chuang, I. L. Quantum Computation and Quantum Information (Cambridge University Press, 2010).

55. Kubo, R. Generalised cumulant expansion method. J. Phys. Soc. Japan 17, 1100-1120 (1962).

56. Kubo, R. Stochastic Liouville equations. J. Math. Phys. 4, 174-183 (1963).

57. Aliferis, P. \& Cross, A. W. Subsystem fault tolerance with the Bacon-Shor code. Phys. Rev. Lett. 98, 220502 (2007).

58. Aliferis, P., Gottesman, D. \& Preskill, J. Accuracy threshold for postselected quantum computation. Quantum Info. Comput. 8, 181-244 (2008).

59. Aharonov, D. \& Ben-Or, M. Fault-tolerant quantum computation with constant error rate. SIAM J. Comput. 38, 1207-1282 (2008).

60. Paz-Silva, G. A., Norris, L. M., Beaudoin, F. \& Viola, L. Extending comb-based spectral estimation to multiaxis quantum noise. Phys. Rev. A 100, 042334 (2019).

61. Hanson, R. \& Burkard, G. Universal set of quantum gates for double-dot spin qubits with fixed interdot coupling. Phys. Rev. Lett. 98, 050502 (2007).

62. Davies, J. The Physics of Low-dimensional Semiconductors (Cambridge University Press, 1997).

63. Annavarapu, R. N. Singular value decomposition and the centrality of Löwdin orthogonalizations. Am. J. Comput. Appl. Math. 3, 33-35 (2013).

64. Zhang, C., Yang, X. C. \& Wang, X. Leakage and sweet spots in triple-quantum-dot spin qubits: a molecular-orbital study. Phys. Rev. A 97, 042326 (2018).

\section{ACKNOWLEDGEMENTS}

M.K.F. was supported by a Singapore Ministry of Education AcRF Tier 1 grant (RG177/ 16), and acknowledges useful discussions with Jun Yoneda. L.H.Z. was supported by the SGUnited program (CP0002392). We thank the Nanyang Technological University (NTU) High Performance Computing Center for computing support, and the Division of Physics and Applied Physics, School of Physical and Mathematical Sciences, NTU, for financial support.

\section{AUTHOR CONTRIBUTIONS}

T.S.K. and M.K.F. contributed to the idea of capacitive two-qubit gates. M.K.F. and L.H.Z. performed the numerical simulations and analytical calculations. All authors analyzed the data and results. T.S.K. wrote the main manuscript with input from all authors. M.K.F. and L.H.Z. wrote the supplementary material. The project was carried out under the supervision of T.S.K.

\section{COMPETING INTERESTS}

The authors declare no competing interests.

ADDITIONAL INFORMATION

Supplementary information The online version contains supplementary material available at https://doi.org/10.1038/s41534-021-00449-4.

Correspondence and requests for materials should be addressed to T.S.K.

Reprints and permission information is available at http://www.nature.com/ reprints

Publisher's note Springer Nature remains neutral with regard to jurisdictional claims in published maps and institutional affiliations.

, corrected publication 2021

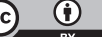

Open Access This article is licensed under a Creative Commons Attribution 4.0 International License, which permits use, sharing, adaptation, distribution and reproduction in any medium or format, as long as you give appropriate credit to the original author(s) and the source, provide a link to the Creative Commons license, and indicate if changes were made. The images or other third party material in this article are included in the article's Creative Commons license, unless indicated otherwise in a credit line to the material. If material is not included in the article's Creative Commons license and your intended use is not permitted by statutory regulation or exceeds the permitted use, you will need to obtain permission directly from the copyright holder. To view a copy of this license, visit http://creativecommons. org/licenses/by/4.0/.

๑) The Author(s) 2021, corrected publication 2021 